\title{
The chemical composition of TS 01, the most oxygen-deficient planetary nebula
}

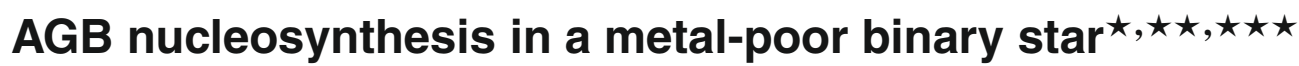

\author{
G. Stasińska ${ }^{1}$, C. Morisset ${ }^{2}$, G. Tovmassian ${ }^{3}$, T. Rauch ${ }^{4}$, M. G. Richer ${ }^{3}$, M. Peña ${ }^{2}$, R. Szczerba ${ }^{5}$, T. Decressin ${ }^{6}$, \\ C. Charbonnel ${ }^{7}$, L. Yungelson ${ }^{8}$, R. Napiwotzki ${ }^{9}$, S. Simón-Díaz ${ }^{10}$, and L. Jamet ${ }^{1}$ \\ ${ }^{1}$ LUTH, Observatoire de Paris, CNRS, Université Paris Diderot, Place Jules Janssen, 92190 Meudon, France \\ e-mail: grazyna.stasinska@obspm.fr \\ 2 Instituto de Astronomia, Universidad Nacional Autonoma de Mexico, Apdo. Postal 70264, Mexico D.F., 04510 Mexico \\ 3 Instituto de Astronomia, Universidad Nacional Autonoma de Mexico, Apdo. Postal 877, Ensenada, Baja California, 22800 Mexico \\ 4 Institute for Astronomy and Astrophysics, Kepler Center for Astro and Particle Physics, Eberhard Karls University, Sand 1, \\ 72076 Tübingen, Germany \\ 5 N. Copernicus Astronomical Center, Rabiańska 8, 87-100 Toruń, Poland \\ ${ }^{6}$ Argelander Institute for Astronomy (AIfA), Auf dem Hügel 71, 53121 Bonn, Germany \\ 7 Geneva Observatory, University of Geneva, ch. des Maillettes 51, 1290 Sauverny, Switzerland and Laboratoire d'Astrophysique \\ de Toulouse-Tarbes, CNRS UMR 5572, Université de Toulouse, 14, Av. E.Belin, 31400 Toulouse, France \\ 8 Institute of Astronomy of the Russian Academy of Sciences, 48 Pyatniskaya Str., 119017 Moscow, Russia \\ 9 Centre for Astrophysics Research, University of Hertfordshire, College Lane, Hatfield AL109AB, UK \\ 10 Instituto de Astrofísica de Canarias, 38200 La Laguna, Tenerife, Spain
}

Received 30 April 2009 / Accepted 15 December 2009

\section{ABSTRACT}

The planetary nebula TS 01 (also called PN G 135.9+55.9 or SBS 1150+599A) with its record-holding low oxygen abundance and its double degenerate close binary core (period $3.9 \mathrm{~h}$ ) is an exceptional object located in the Galactic halo.

We have secured observational data in a complete wavelength range to pin down the abundances of half a dozen elements in the nebula. The abundances are obtained via detailed photoionization modelling which takes into account all the observational constraints (including geometry and aperture effects) using the pseudo-3D photoionization code Cloudy_3D. The spectral energy distribution of the ionizing radiation is taken from appropriate model atmospheres. Incidentally we find from the new observational constraints that both stellar components contribute to the ionization: the "cool" one provides the bulk of hydrogen ionization, while the "hot" one is responsible for the presence of the most highly charged ions, which explains why previous attempts to model the nebula experienced difficulties.

The nebular abundances of $\mathrm{C}, \mathrm{N}, \mathrm{O}$, and $\mathrm{Ne}$ are found to be $1 / 3.5,1 / 4.2,1 / 70$, and $1 / 11$ of the solar value respectively, with uncertainties of a factor 2. Thus the extreme $\mathrm{O}$ deficiency of this object is confirmed. The abundances of $\mathrm{S}$ and Ar are less than 1/30 of solar. The abundance of $\mathrm{He}$ relative to $\mathrm{H}$ is $0.089 \pm 0.009$.

Standard models of stellar evolution and nucleosynthesis cannot explain the abundance pattern observed in the nebula. To obtain an extreme oxygen deficiency in a star whose progenitor has an initial mass of about $1 M_{\odot}$ requires an additional mixing process, which can be induced by stellar rotation and/or by the presence of the close companion. We have computed a stellar model with an initial mass of $1 M_{\odot}$, appropriate metallicity, and initial rotation of $100 \mathrm{~km} \mathrm{~s}^{-1}$, and find that rotation greatly improves the agreement between the predicted and observed abundances.

Key words. planetary nebulae: individual: TS 01 - ISM: abundances - stars: AGB and post-AGB - binaries: general nuclear reactions, nucleosynthesis, abundances

\footnotetext{
* Based on observations obtained at the Canada-France-Hawaii Telescope (CFHT) which is operated by the National Research Council of Canada, the Institut National des Sciences de l'Univers of the Centre National de la Recherche Scientifique of France, and the University of Hawaii.

$\star \star$ Based on observations with the NASA/ESA Hubble Space Telescope, obtained at the Space Telescope Science Institute, which is operated by the Association of Universities for Research in Astronomy, Inc., under NASA contract NAS 5-26555.

$\star \star \star$ Based on observations made with the Spitzer Space Telescope, which is operated by the Jet Propulsion Laboratory, California Institute of Technology, under NASA contract 1407.
}

\section{Introduction}

SBS 1150+599A was discovered in the second Byurakan Sky Survey and first classified as a cataclysmic variable (Stepanian et al. 1999). Tovmassian et al. (2001) discussed in detail the nature of the object and arrived at the conclusion that it is in fact a planetary nebula (PN). The object was renamed PNG 135.9+55.9, following the nomenclature for Galactic PNe from the Strasbourg-ESO catalogue of Galactic Planetary Nebulae (Acker et al. 1992). For the sake of brevity we will refer to it as TS 01 in the rest of the paper. This PN is special in at least three important aspects. First of all, its oxygen abundance is very low, significantly lower than in any other PN known up 
to now (Tovmassian et al. 2001; Richer et al. 2002; Jacoby et al. 2002; Péquignot \& Tsamis 2005). Second, its nucleus is a spectroscopic binary with a period of only a few hours (Tovmassian et al. 2004). Third, it appears from estimates of the nature and masses of the two stellar components, that TS 01 could turn into a double degenerate type Ia supernova (Tovmassian et al. 2004). Each of these aspects, even taken alone, makes TS 01 an exceptional object.

In this paper, we reexamine the chemical composition of TS 01. Briefly, the story of the determination of the chemical composition of this object is the following. Tovmassian et al. (2001) had optical spectra of TS 01 in the range 3900-7000 obtained with $2 \mathrm{~m}$ class telescopes which showed no lines from heavy elements except a very weak [O III] $\lambda 5007$, with an intensity a few percent of $\mathrm{H} \beta$. A coarse photoionization analysis suggested an oxygen abundance smaller than 1/100 solar. Note that standard empirical methods for abundance determinations in PNe cannot be used for TS 01 , since the electron temperature cannot be determined directly from observations. To go further in the abundance determination of TS 01 required an estimate of the effective temperature of the central star. One way is to obtain a good blue spectrum of the PN, and use the [Ne V] $23426 /$ [Ne III] $\lambda 3869$ ratio (or a limit on it) as a constraint. Richer et al. (2002) at the Canada-France-Hawaii Telescope (CFHT) and Jacoby et al. (2002) at the Multiple Mirror Telescope (MMT) secured deep blue spectra to detect these lines. Jacoby et al. (2002) detected the [Ne V] $\lambda 3426$ line at a level of $0.8 \mathrm{H} \beta$. Richer et al. (2002) found only an upper limit of $0.1 \mathrm{H} \beta$ ! Concerning the [Ne III] $\lambda 3869$ line, Jacoby et al. (2002) measured an intensity about ten times higher than Richer et al. (2002). The two papers appeared within a few days of each other on astro-ph, revealing this big conflict in the observations. The two groups conducted independent photoionization analyses, and both concluded that the $\mathrm{O} / \mathrm{H}$ ratio is less than $1 / 100$ solar (the main reason for their similar result for the oxygen abundance was the similar [Ne v] $\lambda 3426 /[\mathrm{Ne}$ III] $\lambda 3869$ ratio used by both studies). Péquignot \& Tsamis (2005) made a combination of the two observational data sets and conducted their own photoionization analysis. They concluded that the $\mathrm{O} / \mathrm{H}$ ratio of TS 01 lies between 1/30-1/15 solar (still holding the record for the most oxygen poor planetary nebula, but much higher than previously published). However, Péquignot \& Tsamis (2005) neglected to consider observations of TS 01 made with the Hubble Space Telescope (HST) and the Far Ultraviolet Spectroscopic Explorer (FUSE). As a result, some of their "predicted" line intensities are in conflict with what is actually observed in the UV. HST observations were obtained in 2003 and presented in a short, preliminary version by Jacoby et al. (2006). Those authors quoted an oxygen abundance of 1/30-1/40 solar, and carbon and nitrogen abundances roughly $1 / 10$ solar.

Before embarking on a new determination of abundances, we have chosen to gather the best possible observations at all wavelength ranges. These data provide many more constraints than were available in any previous study. In order to make the best use of the large amount of data obtained with different telescopes, we use a pseudo-3D photoionization code, Cloudy_3D, which is able to account for the nebular geometry as we see it now, and with which we can properly take into account the aperture effects. This code is based on CLOUDY (Ferland et al. 1998) and was written by Morisset (2006).

The paper is organized as follows. Section 2 presents the new observational material: several optical spectra, HST imaging and spectroscopy, infrared spectroscopy with the Spitzer Telescope, and mentions our X-ray observations with XMM. Section 3
Table 1. Log and characteristics of the spectroscopic observations.

\begin{tabular}{llllc}
\hline \hline Telescope & Date & $\lambda$ Range & Resolution & Aperture \\
\hline FUSE & 30 Jan. 02 & $900-1200 \AA$ & & $30 \times 30^{\prime \prime}$ \\
HST STIS & 4 May 03 & $1170-1700 \AA$ & $1.20 \AA$ & $0.5^{\prime \prime}$ \\
CFHT MOS & 1 May 03 & $3400-5300 \AA$ & $3.0-3.5 \AA$ & $1^{\prime \prime}$ \\
CFHT MOS & 4 Mar. 01 & $3400-8000 \AA$ & $23 \AA$ & $5^{\prime \prime}$ \\
Kitt Peak & 1 Jan. 03 & $3600-7500 \AA$ & $\AA$ & $2^{\prime \prime}$ \\
SDSS & & $3819-9196 \AA$ & $2-4 \AA$ & diameter $3^{\prime \prime}$ \\
Spitzer IRS SH & 22 Apr. 06 $9.9-19.6 \mu \mathrm{m}$ & 600 & $4.7 \times 11.3^{\prime \prime}$ \\
Spitzer IRS LH 22 Apr. 06 & $18.7-37.2 \mu \mathrm{m}$ & 600 & $11.1 \times 22.3^{\prime \prime}$ \\
\hline
\end{tabular}

summarizes other data that we used as constraints for the photoionization modelling. Section 4 describes our modelling strategy and presents our "reference model". Section 5 evaluates the error bars on the derived elemental abundances, taking into account observational uncertainties in emission-line fluxes, uncertainties in model input parameters and also uncertainties arising from an imperfect description of the physical processes included in the models. In Sect. 6, we compare the chemical composition of TS 01 with that of other PNe in the Galactic halo and discuss it in terms of stellar nucleosynthesis in the asymptotic giant branch (AGB) phase. Finally, Sect. 7 summarizes our main findings.

\section{New observational data on emission lines}

We present the observational data that we secured on TS 01 and its stellar core since the work presented in Tovmassian et al. (2004). Some of those data were already briefly reported in conference proceedings, but here we describe the acquisition and reduction processes in more detail. Note that the observations and reductions were done by different people and at different epochs, when our knowledge on the object was not the same. This explains the differences in the tactics employed to reduce the data, estimate the line fluxes and correct for reddening. We did not try to fully homogenize the data reduction process, since we felt it unnecessary for our purposes.

The log and characteristics of each set of observations are given in Table 1.

\subsection{Imaging}

We (M.P.) retrieved the data corresponding to the proposal ID 9466 from the HST archives and analyzed them. The observations were performed on May 5, 2003. Two types of data are available: direct imaging and spectroscopy.

Direct imaging was obtained with the Advanced Camera for Surveys (ACS). The high resolution channel with a field of view of $26^{\prime \prime} \times 29^{\prime \prime}$ and a plate-scale of $0.027^{\prime \prime}$ per pixel, with filters around $\mathrm{H} \alpha$ (central wavelength $6581.97 \pm 162.8 \AA$ ) and [Ne v] $\lambda 3426$ (central wavelength $3432.8 \pm 42.66 \AA$ ) were used.

Figure 1 shows the $\mathrm{H} \alpha$ image obtained by averaging the four calibrated frames j8do01021, j8do01022, j8do01023, j8do01024 (870 s exposure time each; $58 \mathrm{~min}$ in total), after aligning them with respect to j8do01021. The image is roughly elliptical in shape with two brighter, symmetrically-placed lobes at a position angle of about $103^{\circ}$ that extend the full major axis of the ellipse. The nebula is not perfectly symmetric, with the outermost southern part much fainter. The size of the nebular image is about $5^{\prime \prime}$.

Figure 2 shows the same image as Fig. 1, with the different observing apertures indicated: continuous lines for Kitt Peak, 


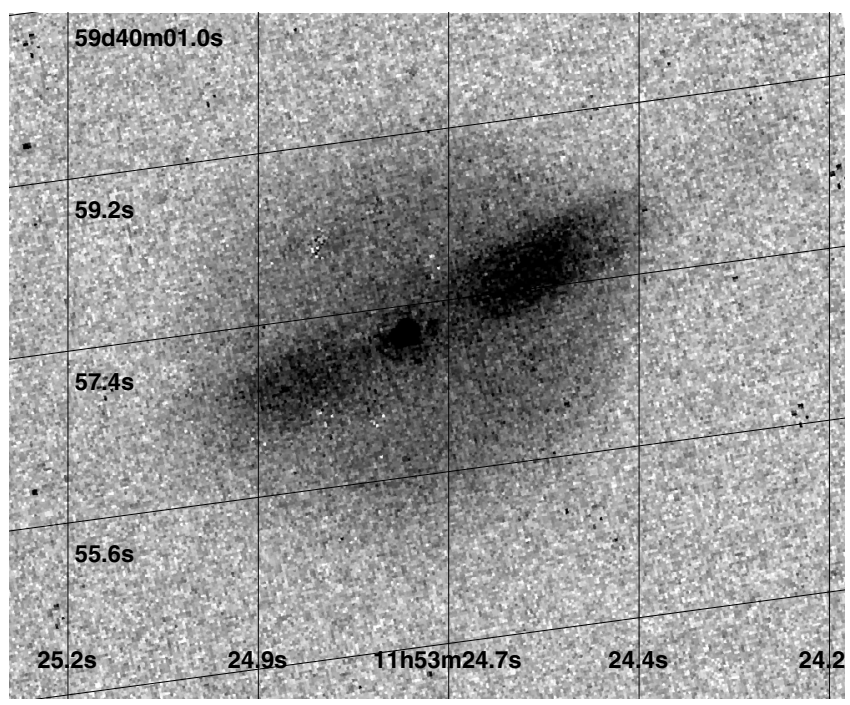

Fig. 1. HST-ACS H $\alpha$ image of TS 01, with a logarithmic grey scale. A grid with the position scale (RA and Dec) is traced. North is up.

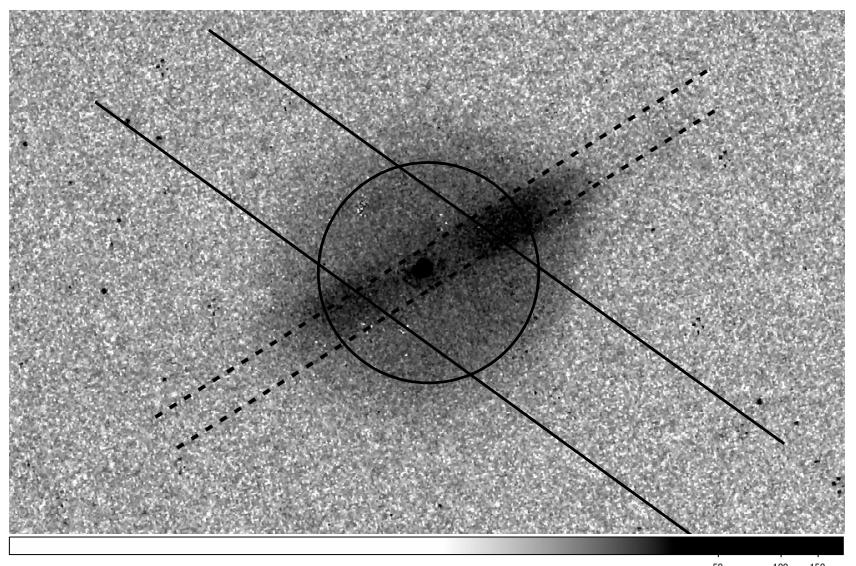

Fig. 2. HST-ACS $\mathrm{H} \alpha$ image of TS 01. The locations of the different spectroscopic apertures are indicated: Kitt Peak (continuous lines), HST STIS (dotted lines), SDSS (circle). See text for the CFHT 2003 observations.

dotted lines for HST STIS, circle for SDSS. For the CFHT 2003 observations, the slit was rotated before each of the seven exposures (see Sect. 2.2.1), so as to remain as close as possible to the parallactic angle. The position of the slit is not indicated in the figure for the sake of clarity, but was taken into account correctly when comparing the predicted line intensities with the observed ones (see Sect. 4.2).

Figure 3 shows an average of the $[\mathrm{Ne} \mathrm{v}] \lambda 3432$ calibrated images j8do01011, j8do01012, j8do01013 and j8do01014 (3000 s of exposure time each; $200 \mathrm{~min}$ in total). This image reveals a very faint, roughly spherical extended nebulosity and an important emission in the centre, probably caused by the stellar core. Some faint extensions are marginally detected in the directions of the $\mathrm{H} \alpha$ lobes.

\subsection{Optical spectroscopy}

\subsubsection{CFHT data}

CFHT 2003 TS 01 was reobserved at the CFHT by M.R. and G.S. on 1 May 2003 using the MOS spectrograph and a 1" slit (Le Fèvre et al. 1994). The U900 grism was used, giving a

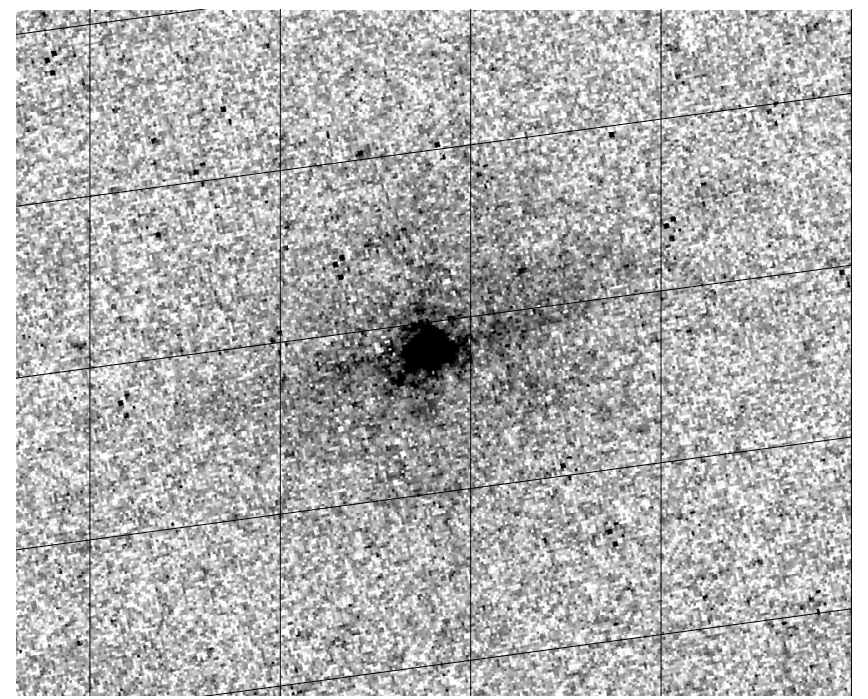

Fig. 3. HST-ACS image of TS 01 in the [Ne v] $\lambda 3426$ line, on a logarithmic grey scale. Same orientation and scale as Fig. 1.

spectral range of $3400-5300 \AA$ and a spectral resolution of 3-3.5 A (measured from arc lamp spectra). Seven 1800 s exposures were obtained. During each exposure, the slit was set to within $10^{\circ}$ of the parallactic angle. Details of the reduction process of the individual exposures are given in Tovmassian et al. (2004).

To obtain a high signal-to-noise spectrum of the nebular emission lines, it is necessary to account for the stellar and nebular continuum emission. These contributions were subtracted from the individual exposures before summing the individual spectra. First, the observed spectra were shifted in velocity so that the stellar absorption line was at rest. Next, the $\mathrm{H} \beta$ intensity was measured and used to scale a model of the nebular continuum emission. Since the $\mathrm{H} \beta$ flux is affected by stellar absorption, the stellar absorption was assumed to have an equivalent width of $13 \AA$, a value typical for the models used (see below). This scaled nebular continuum was then subtracted from the observed spectrum. Then, a model stellar atmosphere was scaled so as to match the observed continuum level and subtracted from the observed spectrum. This procedure leaves a pure nebular emission line spectrum, supposing that the model nebular and stellar continua are representative of their real counterparts. It is unlikely that subtracting the continua introduces significant uncertainty into our final line intensities. Model stellar spectra with $\left(T_{\mathrm{eff}}, \log g\right)$ pairs of $(90 \mathrm{kK}, 5.05),(120 \mathrm{kK}, 5.35)$, and $(150 \mathrm{kK}$, 5.56) computed by R.N. (see Tovmassian et al. 2004) were subtracted from our observed spectra and the differences in the resulting line intensities were always smaller than the uncertainties in the fits ${ }^{1}$.

Once the stellar and nebular continua were subtracted, the individual nebular spectra were shifted back to their original velocities and summed. We measured the nebular emission line strengths from this final spectrum. The line intensities were measured using INTENS, a locally-implemented software package (McCall et al. 1985). This software simultaneously fits a sampled Gaussian function to the emission line(s) and a straight

\footnotetext{
1 This treatment was applied well before we had understood that the optical continuum was dominated by a star of $55 \mathrm{kK}$ (see Sect. 3.3). In view of the fact that the lines we use for the dignostics discussed in the present paper are hardly affected by this correction, we decided not to redo the subtraction using more adequate model stellar spectra.
} 


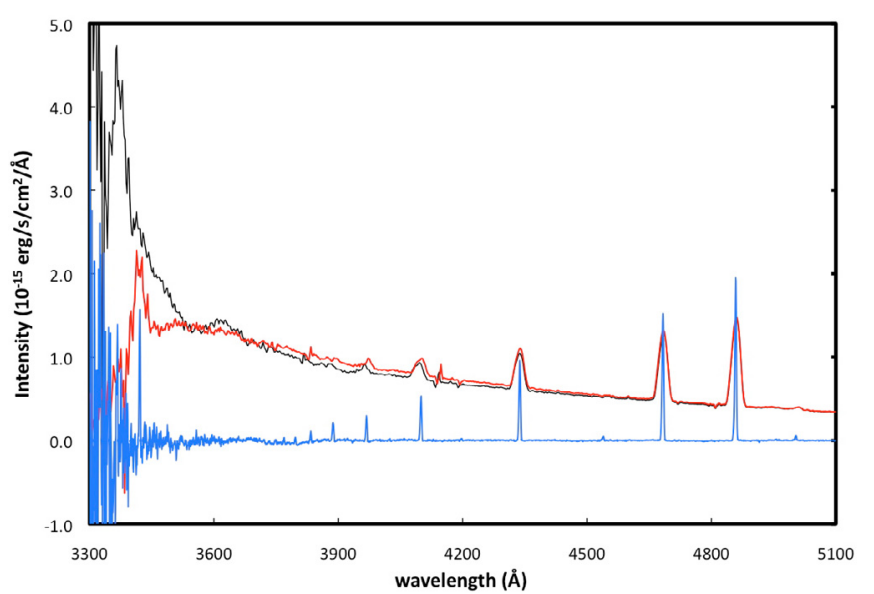

Fig. 4. Flux-calibrated CFHT spectra of TS01. In blue is the CFHT 2003 spectrum with the nebular and stellar continua subtracted, in black the original CFHT 2001 spectrum, and in red the CFHT 2001 spectrum with the correct wavelength solution.

line to the continuum. It returns the line strengths, line wavelengths, and uncertainties in these quantities. The line intensities presented in Table 2 together with their uncertainties are those measured after subtracting the stellar spectrum for $T_{\text {eff }}=120 \mathrm{k} \mathrm{K}$ and $\log g=5.35$. The listed intensities are not corrected for reddening. In the case of no detection, two-sigma upper limits are given instead.

We note that the $[\mathrm{Ne} \mathrm{V}] \lambda 3426$ line is present, and strong. Its intensity is of the same order as in the spectrum of Jacoby et al. (2002), and much higher than the upper limit given by Richer et al. (2002). The remaining lines have intensities roughly in agreement with those published by Richer et al. (2002) and Jacoby et al. (2002), except for the [Ne III] line which appeared on the top of a bump in Jacoby et al. (2002) and was attributed a high intensity in that paper.

CFHT 2001 In view of the important discrepancy with the Richer et al. (2002) data concerning the [Ne v] $\lambda 3426$ line, we decided to reanalyze the spectrum of TS 01 we had obtained in March 2001 at the CFHT. First, though, we refer the interested reader to Richer et al. (2002) for a discussion of the details of these observations. The basic difficulty with these observations was that the arc lamp spectra were taken with the same $5^{\prime \prime}$ slit used for the object observations, which resulted in an arc spectrum with severely blended lines. After repeating the wavelength calibration more carefully, we found that our previous solution had stretched out the spectrum at the shortest wavelengths, leading us to not recognize the [Ne v] $\lambda 3426$ line because of its low contrast with respect to the continuum and its erroneous wavelength. The analysis of this spectrum was considerably simpler given that the stellar features were not resolved. We simply fit the continuum shape and removed it (INTENS assumes that the continuum is a straight line), then measured the line intensities with INTENS. The resulting line intensities are given in Table 2. The intensity we find now for [Ne V] $\lambda 3426$ agrees with the one given by Jacoby et al. (2002) and is compatible within two sigmas with the one obtained with the CFHT 2003 data. Slit effects could perhaps explain the slight difference in intensity between CFHT 2001 and CFHT 2003.
Table 2. Intensities of optical lines, corrected for stellar absorption, but not for reddening, with respect to $\mathrm{H} \beta=100$.

\begin{tabular}{|c|c|c|c|c|}
\hline & SDSS & Kitt Peak & CFHT 2003 & CFHT 2001 \\
\hline$[\mathrm{Ne} \mathrm{V}] 3426$ & & & $55.54 \pm 5.19$ & $84.05 \pm 13.07$ \\
\hline [O II] 3727 & & & $<0.60$ & $<1.55$ \\
\hline H I 3735 & & & $1.07 \pm 0.31$ & \\
\hline H I 3751 & & $4.94 \pm 1.18$ & $1.70 \pm 0.32$ & \\
\hline H I 3772 & & $4.36 \pm 1.30$ & $2.30 \pm 0.35$ & \\
\hline H I 3798 & & $<3.0$ & $2.64 \pm 0.38$ & \\
\hline H I 3836 & $6.73 \pm 0.53$ & $3.71 \pm 0.82$ & $4.67 \pm 0.37$ & \\
\hline [Ne III] 3869 & $<1.2$ & $<1.5$ & $0.77 \pm 0.31$ & $1.67 \pm 0.48$ \\
\hline H I 3889 & $9.06 \pm 0.43$ & $7.81 \pm 0.87$ & $9.28 \pm 0.45$ & $2.37 \pm 0.47$ \\
\hline H I 3970 & $13.29 \pm 0.44$ & $13.24 \pm 0.79$ & $12.70 \pm 0.47$ & $5.85 \pm 0.58$ \\
\hline He II 4027 & $<1.2$ & $<1.5$ & $0.56 \pm 0.21$ & \\
\hline C III 4069 & $2.1::$ & $<1.5$ & $<0.58$ & \\
\hline H I 4102 & $25.11 \pm 0.42$ & $25.49 \pm 0.62$ & $25.16 \pm 0.43$ & $19.79 \pm 1.06$ \\
\hline He II 4201 & $<1.2$ & $<1$ & $1.04 \pm 0.19$ & \\
\hline C II 4267 & $<1.2$ & $<1$ & $<0.32$ & \\
\hline H I 4340 & $46.09 \pm 0.38$ & $48.06 \pm 0.44$ & $47.19 \pm 0.50$ & $42.49 \pm 0.80$ \\
\hline [O III] 4363 & $<1.2$ & $<1$ & $<0.41$ & \\
\hline N III 4379 & $<1.2$ & $<1$ & $<0.09$ & \\
\hline He I 4471 & $<1.2$ & $<1$ & $<0.11$ & \\
\hline He II 4543 & $2.97 \pm 0.33$ & $2.91 \pm 0.35$ & $2.46 \pm 0.22$ & \\
\hline [Ar V] 4626 & $<1.2$ & $<1$ & $<0.38$ & \\
\hline O IV 4632 & $<1.2$ & $<1$ & $<0.38$ & \\
\hline C III 4650 & $<1.2$ & $<1$ & $<0.38$ & \\
\hline C IV 4659 & $<1.2$ & $<1$ & $<0.38$ & \\
\hline C IV 4659 & $<1.2$ & $<1$ & $<0.24$ & \\
\hline He II 4686 & $75.13 \pm 0.31$ & $79.02 \pm 0.28$ & $77.50 \pm 0.95$ & $79.06 \pm 1.11$ \\
\hline [Ar IV] 4711 & $<1.2$ & $<1$ & $<0.15$ & \\
\hline [Ne IV] 4715 & $<1.2$ & $<1$ & $0.32 \pm 0.08$ & \\
\hline [Ne IV] 4725 & $<1.2$ & $<1$ & $0.26 \pm 0.08$ & \\
\hline [Ar IV] 4740 & $<1.2$ & $<1$ & $<0.15$ & \\
\hline $\mathrm{H} \beta 4861$ & $100.00 \pm 0.29$ & $100.00 \pm 0.26$ & $100.00 \pm 0.78$ & $100.00 \pm 1.59$ \\
\hline O V 4930 & $<1.2$ & $<0.8$ & $<0.28$ & \\
\hline N V 4945 & $<1.2$ & $<0.8$ & $<0.28$ & \\
\hline [O III] 4959 & $<1.2$ & $<0.99 \pm 0.29$ & $0.59 \pm 0.13$ & $0.69 \pm 0.69$ \\
\hline [O III] 5007 & $1.82 \pm 0.28$ & $2.16 \pm 0.26$ & $2.53 \pm 0.16$ & $1.81 \pm 0.79$ \\
\hline$[\mathrm{Fe} \mathrm{VI}] 5146$ & $<10.0$ & $<0.8$ & $<0.28$ & \\
\hline He II 5411 & $5.8 \pm 0.22$ & $6.49 \pm 0.28$ & & $5.12 \pm 0.31$ \\
\hline He I 5876 & $<0.8$ & $<0.8$ & & $<0.19$ \\
\hline [Fe VII] 6087 & $<0.8$ & $<0.8$ & & \\
\hline $\mathrm{H} \alpha 6563$ & $322.03 \pm 0.15$ & $306.38 \pm 0.22$ & & $248.54 \pm 2.63$ \\
\hline [N II] 6584 & $<1.6$ & & & \\
\hline [S II] 6716 & $<1.6$ & & & \\
\hline [S II] 6731 & $<1.6$ & & & \\
\hline [Ar V] 7006 & $<1.6$ & & & \\
\hline He I 7065 & $<1.6$ & & & \\
\hline [Ar III] 7136 & $<1.6$ & & & \\
\hline [O II] 7320 & $<1.6$ & & & \\
\hline [O II] 7330 & $<1.6$ & & & \\
\hline [O II] 7333 & $<1.6$ & & & \\
\hline ?? 7408 & $0.82 \pm 0.24$ & & & \\
\hline [Cl IV] 7530 & $<1.6$ & & & \\
\hline He II 7600 & $<1.6$ & & & \\
\hline O V 7611 & $<1.6$ & & & \\
\hline O IV 7713 & $<1.6$ & & & \\
\hline C IV 7726 & $<1.6$ & & & \\
\hline [Ar III] 7751 & $<1.6$ & & & \\
\hline [Cl IV] 8046 & $<1.6$ & & & \\
\hline CIII 8196 & $<1.6$ & & & \\
\hline He II 8237 & $1.76 \pm 0.16$ & & & \\
\hline H P16 8502 & $<1.6$ & & & \\
\hline H P15 8545 & $<1.6$ & & & \\
\hline H P14 8598 & $<1.6$ & & & \\
\hline H P13 8665 & $<1.6$ & & & \\
\hline H P12 8750 & $1.29 \pm 0.26$ & & & \\
\hline H P10 9014 & $1.7:$ & & & \\
\hline [S III] 9069 & $<1.6$ & & & \\
\hline
\end{tabular}




\subsubsection{Kitt Peak data}

TS 01 was observed at the $4 \mathrm{~m}$ telescope of Kitt Peak National Observatory on 1 January 2003. The grating used was KPC-10A, and the slit $2^{\prime \prime} \times 300^{\prime \prime}$, with an orientation of PA $=44.7^{\circ}$. Two exposures of $600 \mathrm{~s}$ were obtained. The data were reduced by L.J., employing the same procedure as for the SDSS spectrum, explained below.

\subsubsection{SDSS and Kitt Peak data}

The spectrum of TS 01 appears in the data of the Sloan Digital Sky Survey SDSS (http://www.sdss.org) under the name 0953-52411-1602. We present its analysis performed by L.J.

We separated the nebular emission from the stellar spectrum and evaluated the reddening with as few free parameters as possible. We assumed the stellar spectrum to be that of a single white dwarf (WD), hence neglecting the possible contribution of the companion; we considered three model WD spectra at temperatures of 90, 120 and $150 \mathrm{kK}$ (the same as used for the CFHT 2003 spectrum). As for the nebular continuum, we computed the free-free and free-bound emissivities of $\mathrm{H}^{+}$and $\mathrm{He}^{++}$with the CHIANTI code (Landi et al. 2006), assuming an abundance ratio $\mathrm{He}^{++} / \mathrm{H}^{+}=0.075$ and an electronic temperature of $30 \mathrm{kK}$ (Richer et al. 2002). We also retrieved the $\mathrm{H} \gamma$ nebular emissivity at this temperature from Storey \& Hummer (1995).

First, we computed a model of the total (stellar+nebular) spectrum around the $\mathrm{H} \gamma$ line. In each of the WD spectral models, the $\mathrm{H} \gamma$ line has a Voigt profile with a given equivalent width (EW) $W_{\mathrm{wd}}$, Gaussian width $\sigma_{\mathrm{wd}}$ and Lorentzian width $a_{\mathrm{wd}}$. As for the nebular emission, we computed the EW $W_{\text {neb }}$ of the emission line with respect to the nebular continuum. Furthermore, we assumed the real width of the nebular line to be much smaller than the instrumental one, so its observed Gaussian and Lorentzian widths, respectively $\sigma_{\text {inst }}$ and $a_{\text {inst }}$, are representative of the instrumental PSF. Consequently, the observed widths of the stellar line are $\sigma_{*}=\left(\sigma_{\mathrm{wd}}^{2}+\sigma_{\text {inst }}^{2}\right)^{1 / 2}$ and $a_{*}=a_{\mathrm{wd}}+a_{\text {inst }}$. We normalized the local stellar+nebular continuum with the fit of a slope on either side of the line. Finally, we let the central wavelengths of the stellar and nebular line, respectively $\lambda_{*}$ and $\lambda_{\text {neb }}$, be independent from each other. Using the data and assumptions gathered, we fitted a consistent model on the observed spectrum around the line. Calling $V_{\text {neb }}(\lambda)$ the profile of the nebular line, $V_{*}(\lambda)$ that of the star (both being normalized to an EW of unity), and $C_{*}$ the stellar contribution to the flux, the model can be written as

$$
\begin{aligned}
F_{\lambda}(\lambda)= & C_{*}\left(1-W_{\mathrm{wd}} V_{*}\left(\lambda-\lambda_{*} ; \sigma_{\mathrm{inst}}, a_{\mathrm{inst}}, \sigma_{\mathrm{wd}}, a_{\mathrm{wd}}\right)\right) \\
& +\left(1-C_{*}\right)\left(1+W_{\mathrm{neb}} V_{\mathrm{neb}}\left(\lambda-\lambda_{\mathrm{neb}} ; \sigma_{\mathrm{inst}}, a_{\mathrm{inst}}\right)\right) .
\end{aligned}
$$

For all three WD model atmospheres, we obtained very good fits with no visible systematic residuals.

Given that the intrinsic $\mathrm{H} \alpha / \mathrm{H} \beta$ nebular line ratio in TS 01 is not merely the case $\mathrm{B}$ recombination value, and given the additional problems with $\mathrm{H} \alpha$ (see Sect. 5.3.1), we cannot use this ratio to evaluate the extinction. Hence, we used the continuum to measure the latter. We first corrected the data for the

\footnotetext{
2 The analysis by L.J. was done soon after our discovery of the spectrum in SDSS data release 2. In data release 6 (Adelman-McCarthy et al. 2008), SDSS spectra were recalibrated, resulting in an increase of about $30 \%$ of the fluxes of TS 01 . Line ratios remained unchanged. Therefore, the analysis of L.J. remains valid. On the other hand, whenever we needed to consider the TS 01 continuum in this paper, we used the recalibrated spectrum.
}

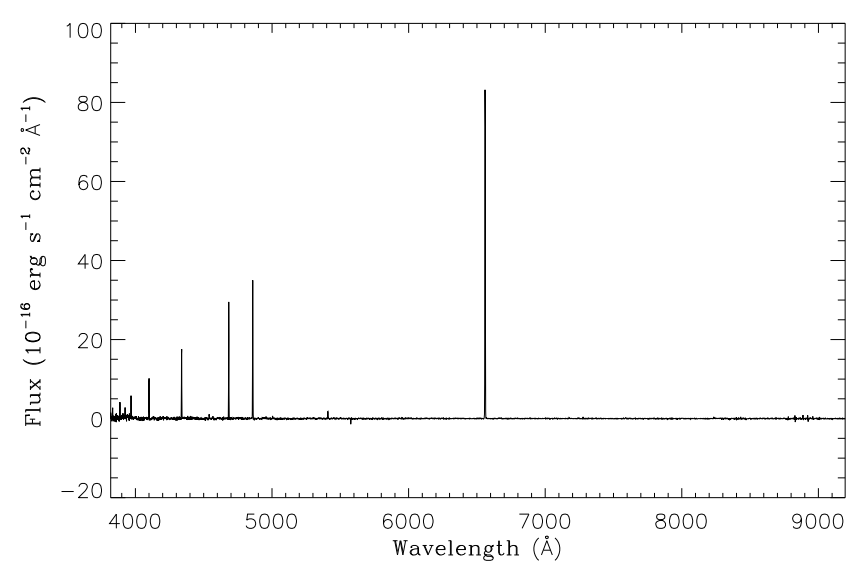

Fig. 5. Fully processed SDSS spectrum (dereddened, free of continuum and stellar lines).

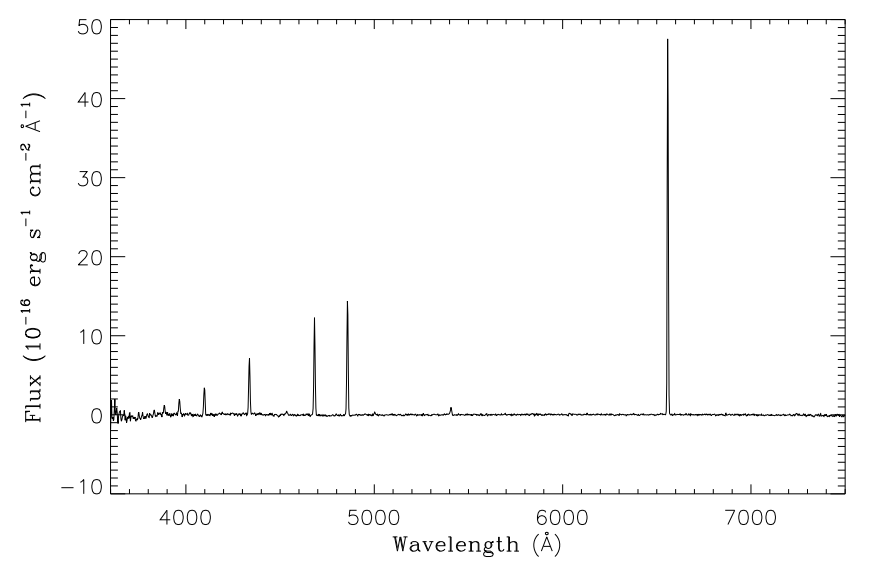

Fig. 6. Fully processed Kitt Peak spectrum (dereddened, free of continuum and stellar lines).

small foreground extinction $(E(B-V)=0.029)$ estimated by Schlegel et al. (1998). Then, we removed most of the nebular or stellar lines from the observations making use of a median filter. Finally, adopting the SMC extinction law (Prévot et al. 1984; Bouchet et al. 1985) and comparing the filtered spectrum with the theoretical continuum, we evaluated the reddening and corrected the data for it. The choice of the extinction law was motivated by the low metallicity of TS 01 . The reddening amounts obtained (additionally to the foreground one) are $E(B-V)=0.033,0.044$ and 0.050 for the 90,120 and $150 \mathrm{kK}$ WD models, respectively.

The last processing of the data was the removal of the stellar and nebular continua, to avoid the contamination of the nebular lines by the underlying stellar features. We used the fit of the $\mathrm{H} \gamma$ line to shift the theoretical stellar spectrum and nebular continuum according to their evaluated radial velocities, convolved them by the average instrumental PSF and subtracted them. Finally, we identified visually the detectable lines and measured their fluxes. The fully processed SDSS spectrum with the $90 \mathrm{kK}$ WD model spectrum removed is presented in Fig. 5, while Fig. 6 shows the result of the processing of the Kitt Peak spectrum. The choice of the WD model had a moderate impact on the evaluation of the line fluxes, of order of $2 \%$ for most of them and $2 \sigma$ in the worst case. The intensities of the SDSS spectrum and the Kitt Peak spectrum are listed in Table 2. 


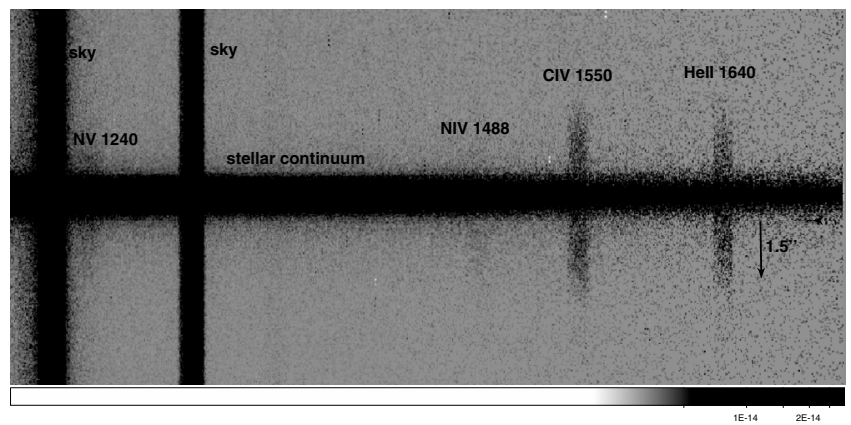

Fig. 7. 2D calibrated HST FUV spectrum of TS 01.

\subsection{Ultraviolet spectroscopy}

\subsubsection{HST STIS data}

The HST STIS spectroscopic data correspond to the same proposal (ID 9466) as the imaging data.

A $52^{\prime \prime} \times 0.5^{\prime \prime}$ slit was used. It was oriented along the bright jet-like emission of the nebula (PA $103^{\circ}$ ), see Fig. 2.

Far UV observations The MAMA detector combined with a G140L grating provided 2D spectra o8do03020, o8do03030, o8do03040, o8do03050 and o8do03060, with 4675 s exposure time each. The spectra cover a wavelength range from 1170 to $1700 \AA$, with a resolving power of 1190 at the central wavelength $1425 \AA$. The $2 \mathrm{D}$ spectra show a bright blue stellar continuum and a few faint and extended emission lines from the nebula. Calibrated 2D spectra were combined (after shifting because the spectroscopic observations were dithered) to produce a 389.6 min spectrum. The resulting $2 \mathrm{D}$ spectrum is shown in Fig. 7. The stellar spectrum shows good signal-to-noise and stellar and interstellar absorption are present. The analysis of the stellar spectrum is presented in a companion paper (Tovmassian et al., in prep.). Regarding nebular lines, the following ones are detected: N V $\lambda 1240, N$ IV] $\lambda 1488$, C IV] $\lambda 1550$ and He II] $\lambda 1640$. Selective absorption of resonance lines by the intervening interstellar medium is treated in Sect. 3.4.

From the combined 2D spectrum the nebular emission was extracted on both sides of the central star, with an extraction window of 60 pixels, equivalent to $1.464^{\prime \prime}$. Figure 8 is a combination of both nebular spectra. The line fluxes in each lobe and the combined values with respect He II] $\lambda 1640$ are listed in Table 3.

Near UV observations The MAMA detector combined with a G230LL grating provided 2D spectra o8do02010, o8do02020, o8do02030, o8do02040 and o8do02050, covering a wavelength range from about 1600 to $3150 \AA$. The calibrated $2 \mathrm{D}$ spectra were combined (after aligning) to produce a spectrum with a total exposure time of $237.5 \mathrm{~min}$. As for the FUV, the NUV stellar spectrum has good signal-to-noise, and stellar and interstellar absorption can be seen. However, no nebular lines are detected. In particular, He II $\lambda 1640, \mathrm{~N}$ III] $\lambda 1750$, and C III] $\lambda 1909$ are not seen. Table 3 gives upper limits for the latter line intensities, with respect to He II $\lambda 1640$, as seen in the FUV spectrum.

\subsubsection{FUSE data}

The observations of TS01 with the Far Ultraviolet Spectroscopic Explorer (FUSE) and their reductions (done by G.T.) are described in Tovmassian et al. (2004). No emission

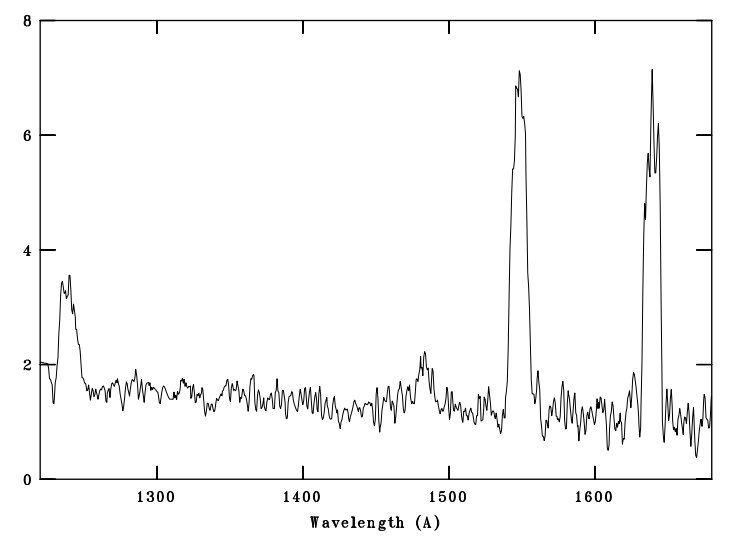

Fig. 8. HST FUV nebular spectrum of TS 01, not corrected for reddening, showing the He II $\lambda 1640 \AA$, N V $\lambda 1240 \AA, N$ IV] $\lambda 1488 \AA$, and C IV $\lambda 1550 \AA$ lines. The fluxes are in $10^{-14} \mathrm{erg} \mathrm{cm}^{-2} \mathrm{~s}^{-1} \AA^{-1}$.

Table 3. Observed HST UV line fluxes, relative to He II 1640.

\begin{tabular}{lccc}
\hline \hline Ion lambda & Bright lobe & Faint lobe & Combined \\
\hline He II $\lambda 1640 \AA$ & $1.56^{a}$ & $1.15^{a}$ & $(2.7+/-0.3)^{a, b}$ \\
N V $\lambda 1240 \AA$ & 0.31 & 0.51 & $0.47+/-0.04$ \\
O IV $\lambda 1402 \AA$ & $<0.06$ & - & $<0.06$ \\
N IV] $\lambda 1488 \AA$ & 0.17 & noisy & $0.12+/-0.05$ \\
C IV $\lambda 1550 \AA$ & 1.10 & 1.33 & $1.28+/-0.10$ \\
N III] $\lambda 1750 \AA$ & $<0.1$ & & $<0.1$ \\
C III] $\lambda 1909 \AA$ & $<0.1$ & & $<0.1$ \\
\hline
\end{tabular}

Notes. ${ }^{(a)}$ The flux of He II $\lambda 1640$ is in units of $10^{-14} \mathrm{erg} \mathrm{cm}^{-2} \mathrm{~s}^{-1}$; ${ }^{(b)}$ all the flux in He II $\lambda 1640$, including both lobes.

lines were detected in the observed wavelength region between 900 and $1200 \AA$, except the $\mathrm{H} \mathrm{Ly} \beta$ line. For the photoionization modelling of the nebula, it is important to determine upper limits to the intensities of nebular lines expected in this wavelength range. We proceeded in the following way. From a previous model of TS 01 we took the computed nebular continuum. We superimposed on it the lines C III $\lambda 977.020$, N III $\lambda 989.799$ and He II $\lambda 992.4$ with $F W H M$ of $0.1 \AA$ (which corresponds to the measured expansion velocity of $30 \mathrm{~km} \mathrm{~s}^{-1}$, see Sect. 3.2). To this we added the central star model mentioned in Sect. 2.2.1. The resulting spectrum was processed through the interstellar hydrogen absorption simulator (http: //violet . pha.jhu .edu/ $\sim$ gak/fwebsim.html) to be compared with the observations. It turned out that the corresponding lines start to be detectable in the resulting spectrum when the line flux reaches approximately $7.5 \times 10^{-14} \mathrm{erg} \mathrm{cm}^{-2} \mathrm{~s}^{-1}$. Indeed, the wavelength region that we are exploiting here is very complicated. Apart from the different interstellar absorptions and terrestrial airglow, the lines in this region also lie at the edges of the detectors where they overlap, and errors are much higher compared to other regions to the red.

\subsection{Mid-infrared spectroscopy}

TS 01 was observed using the infrared spectrograph (IRS, Houck et al. 2004) on board the Spitzer Space Telescope (Werner et al. 2004) on 22 April 2006 (program \#20358). The observations used the short-high (SH: 9.9-19.6 $\mu \mathrm{m} ; R \sim 600$ ) and longhigh (LH:18.7-37.2 $\mu \mathrm{m} ; R \sim 600$ ) modules. The aperture of the SH module is $4.7^{\prime \prime} \times 11.3^{\prime \prime}$ and of the LH one is $11.1^{\prime \prime} \times 22.3^{\prime \prime}$, so the entire nebular flux was measured. The details of the 


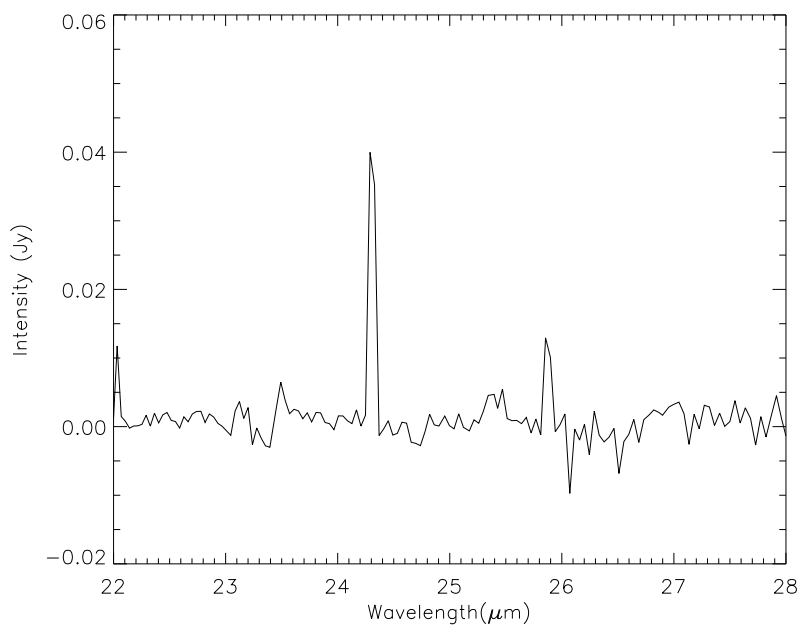

Fig. 9. LH infrared spectrum of TS 01 between 22 and $28 \mu \mathrm{m}$, showing the [O IV] $\lambda 25.89 \mu \mathrm{m}$ and $[\mathrm{Ne} \mathrm{V}] \lambda 24.32 \mu \mathrm{m}$ lines.

performed observations are shown in Table 1. For LH we used four exposure cycles of $240 \mathrm{~s}$ each for on-source and off-source observations, while for $\mathrm{SH}$ only on-source observations were performed with six exposure cycles of $480 \mathrm{~s}$ each. The starting points for our interactive data reduction were the co-added 2D flat-fielded BCD (basic calibrated DATA) images (one for each node position; pipeline version 15.3 for $\mathrm{SH}$ and 17.2 for $\mathrm{LH}$ ). The rogue pixels were removed using the IRSCLEAN tool ${ }^{3}$, with the aggressive parameter equal to 0 . Then the data were processed (full extraction, trimming, defringing and averaging over cycles) into a single spectrum per node position using SMART ${ }^{4}$ (Higdon et al. 2004). A similar procedure has been applied for LH off-source observations, and the obtained spectra have been subtracted from the on-source data for the corresponding node position, to cancel out the sky background. The resulting spectrum between 22 and $28 \mu \mathrm{m}$ is shown in Fig. 9. For the highresolution $\mathrm{SH}$ module no background subtraction was done since no sky measurements were taken and the $\mathrm{SH}$ slit is too small for on-slit background subtraction. Finally, the spectra obtained for both modules were averaged over two node positions, and the detected nebular lines were measured within SMART.

The resulting intensities are listed in Table 4 together with the estimated uncertainties. These uncertainties do not include possible calibration errors. It is generally considered that the absolute flux calibration has an accuracy of 20-30\%. This will be taken into account in the modelling (see Sect. 4.2).

Table 4 also lists the blue-shifts of the lines. One can see that they are consistent with the optical measurements of Tovmassian et al. (2001).

\subsection{XMM data}

TS 01 has been also observed in the X-rays with XMM. The data acquisition and analysis is presented in Tovmassian et al. (in prep.).

\footnotetext{
3 This tool is available from the Spitzer Science Center website: http://ssc.spitzer. caltech. edu.

${ }^{4}$ SMART was developed by the IRS Team at Cornell University and is available through the Spitzer Science Center at Caltech.
}

Table 4. Observed mid IR line fluxes, in units of $10^{-21} \mathrm{~W} \mathrm{~cm}^{-2}$.

\begin{tabular}{lccc}
\hline \hline Ion lambda & $\begin{array}{c}\text { Flux } \\
10^{-21} \mathrm{~W} \mathrm{~cm}^{-2}\end{array}$ & $\begin{array}{c}\text { Uncertainty } \\
10^{-21} \mathrm{~W} \mathrm{~cm}^{-2}\end{array}$ & $\begin{array}{c}\text { Rad. velocity } \\
\mathrm{km} \mathrm{s}^{-1}\end{array}$ \\
\hline$[\mathrm{O} \mathrm{IV}] \lambda 25.89 \mu \mathrm{m}$ & 0.38 & 0.03 & -180 \\
{$[\mathrm{Ne} \mathrm{V}] \lambda 24.32 \mu \mathrm{m}$} & 1.50 & 0.20 & -140 \\
{$[\mathrm{Ne} \mathrm{V}] \lambda 14.32 \mu \mathrm{m}$} & 0.82 & 0.10 & \\
\hline
\end{tabular}

\section{What else do we know about TS 01 and its exciting star?}

\subsection{Extinction}

TS 01 suffers only little extinction. Using the observed $\mathrm{H} \gamma / \mathrm{H} \beta$ and $\mathrm{H} \delta / \mathrm{H} \beta$ ratios, Richer et al. (2002) had found $E(B-V) \sim$ 0.3 mag. However, this estimate was made without considering the underlying stellar absorption in the Balmer lines. Due account for this effect significantly reduces the estimated $E(B-V)$, as noted by Tovmassian et al. (2004). The extinction can also be estimated by considering the spectral energy distribution of the stellar core as observed in the far UV by FUSE. Assuming a temperature of $120 \mathrm{kK}$ for the central star, Tovmassian et al. (2004) obtained a good fit to these observations for $E(B-V)=$ $0.045 \mathrm{mag}$, when using a non-canonical value for $R_{V}$ of 2.3 and the interstellar reddening tables from Fitzpatrick (1999). Such a low value of $R_{V}$ compared to the standard one of 3.1 was considered compatible with the location of TS 01 well outside the galactic disk, since the intervening dust is likely composed of smaller grains than in the spiral arms. However, we now know that the temperature of the star which dominates the UV continuum is much cooler (see Sect. 3.3 and Tovmassian et al., in prep.), implying that a steep reddening law is not needed after all. In the remainder of the paper as well as in Tovmassian et al. (in prep.) we use the Fitzpatrick (1999) reddening law parametrized with $R_{V}=3.1$, and take $E(B-V)=0.03$ mag, which satisfactorily accounts for the observed $\mathrm{H}$ and $\mathrm{He}$ line ratios as well as the observed continuum. Note that the absence of an absorption dip at $2200 \AA$ imposes an upper limit of 0.06 for $E(B-V)$.

\subsection{Expansion velocity}

The expansion velocity of TS 01 has been measured by Richer et al. (2003). This parameter is useful to estimate the expansion cooling in the nebula. It also allows one to have an idea of the nebular dynamical age. We adopt $v_{\exp }=30 \mathrm{~km} \mathrm{~s}^{-1}$.

\subsection{The stellar core}

Our understanding of the stellar core of TS 01 has evolved considerably since the first paper where it was suggested that SBS $1150+599 \mathrm{~A}$ is a high excitation planetary nebula (Tovmassian et al. 2001) with a central star that has an effective temperature above $100000 \mathrm{~K}$. Spectroscopic variations in the course of one single night, reported in Tovmassian et al. (2004), indicated the presence of a double system with a compact star. Photometric observations then unambiguously determined a period of $3.92 \mathrm{~h}$ (Napiwotzki et al. 2005). Analysis of the light curve indicated that the visible star is likely an elongated ellipsoid irradiated by a source of higher energy. It also supported the previous conclusion that the companion must be a (pre-?) white dwarf or a neutron star. Finally, X-ray observations (Tovmassian et al. 2007, 2008) obtained with the XMM-Newton 
satellite directly revealed the light from the companion, which turns out to be a hot compact star! Thus, as will be shown later, the "cool" star is the one visible in the optical and the UV and it provides most of the ionizing photons. But it is the "hot" star which gives rise to the high excitation lines observed in the nebula. The best fit to the total spectral energy distribution of the binary core indicates that the cool component has a temperature $T_{\mathrm{c}} \sim 60 \mathrm{kK}$, while the hot component should have $T_{\mathrm{h}} \sim 170 \mathrm{kK}$. However, the determination of the temperature of the hot component is not very accurate. Note that, in the scenario developped by Tovmassian et al. (in prep.), the hot component is an old white dwarf, which has a $170 \mathrm{kK}$ temperature not because it is still early on its cooling path, but because it was heated by nuclear burning of the accreted material on its surface. For the cool component Tovmassian et al. (in prep.) obtains the following: $T_{\mathrm{c}}=58000 \pm 3000 \mathrm{~K}, \log g_{\mathrm{c}} \sim 5.1$. The lower limit on the temperature is the intrinsic temperature of the star, the upper limit corresponds to the zone heated by irradiation. It is important to note that the cool component is not spherical and has not only an inhomogeneous temperature distribution on its surface but also an uneven gravitational acceleration. Its total luminosity is estimated by Tovmassian et al. (in prep.) to be $L_{\mathrm{c}}=1700 L_{\odot}$ with about $30 \%$ uncertainty. Below we will consider for the sake of simplicity that the cool star is sufficiently well represented by a stellar model atmosphere with $T_{\mathrm{c}}=55 \mathrm{kK}$ and $\log g_{\mathrm{c}} \sim 5.1$, with a total luminosity of $1700 L_{\odot}$.

The abundance analysis performed by T.R. on the cool star gives $12+\log \mathrm{He} / \mathrm{H}=10.95$ and $12+\log \mathrm{C} / \mathrm{H}=7.20$, with an uncertainty of about $0.3 \mathrm{dex}$, and upper limits $12+\log \mathrm{N} / \mathrm{H}<$ 6.92 and $12+\log \mathrm{O} / \mathrm{H}<6.80$.

\subsection{Interstellar absorption of nebular UV lines}

In the course of his stellar atmosphere analysis, T.R. noted that the observed C IV $\lambda 1550 \AA$ and N V $\lambda 1240 \AA$ absorption lines were stronger than predicted by his best models. He suggested that these lines are probably affected by interstellar absorption. In that case, the intensities of the C IV $\lambda 1550 \AA$ and N V $\lambda 1240 \AA$ nebular lines are also affected by absorption. Since these lines are crucial for the determination of the nebular abundances in TS 01, we here explain how we corrected for this effect.

We use the following notations (all quantities are a function of wavelength): $F_{\mathrm{S}}$ : flux extracted at the position of the star; $F_{\mathrm{N}}$ : flux extracted at the adjacent position in the nebula; $F_{*}$ : real stellar flux; $F_{\text {neb }}$ : real nebular flux; $F_{\text {sky }}$ : sky emission and nebular continuum. The optical depth due to interstellar absorption is denoted $\tau$. We have

$F_{\mathrm{S}}=\left(F_{*}+F_{\text {neb }}+F_{\text {sky }}\right) \exp (-\tau)$

and

$F_{\mathrm{N}}=\left(F_{\text {neb }}+F_{\text {sky }}\right) \exp (-\tau)$,

so that, in the spectrum analyzed by R.T., we have:

$F_{\mathrm{S}}-F_{\mathrm{N}}=F_{*} \exp (-\tau)$.

Concerning the C IV $\lambda 1550$ line, reading out from Fig. 10 we find $F_{*} \exp (-\tau)=8.7 \times 10^{-15}$ (black line in the figure), leading to $\exp (-\tau)=0.75$. Therefore, if the measured nebular flux is $F_{\text {neb }} \exp (-\tau)=1.28 \times 2.7 \times 10^{-14}=3.45 \times 10^{-14}$ (last column of Table 3), the nebular flux after correction for absorption is $F_{\text {neb }}=4.65 \times 10^{-14}$ with an uncertainty of about $20 \%$.

Concerning the $\mathrm{N} v \lambda 1240 \AA$ line, we find from Fig. $11 F_{*}=$ $2.1 \times 10^{-14}$ (red line in the figure), $F_{*} \exp (-\tau)=1.9 \times 10^{-14}$

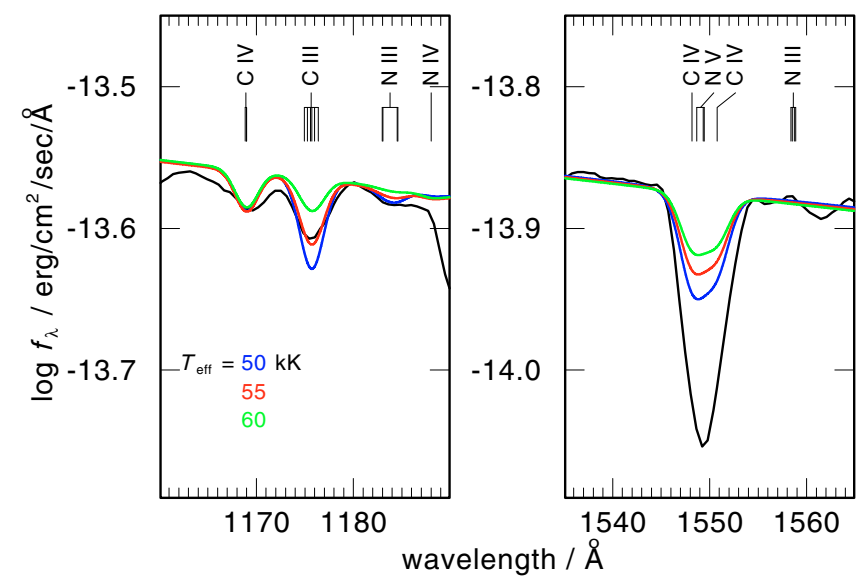

Fig. 10. Stellar model atmosphere fitting of carbon lines in the stellar core of TS 01. Observation is in black.

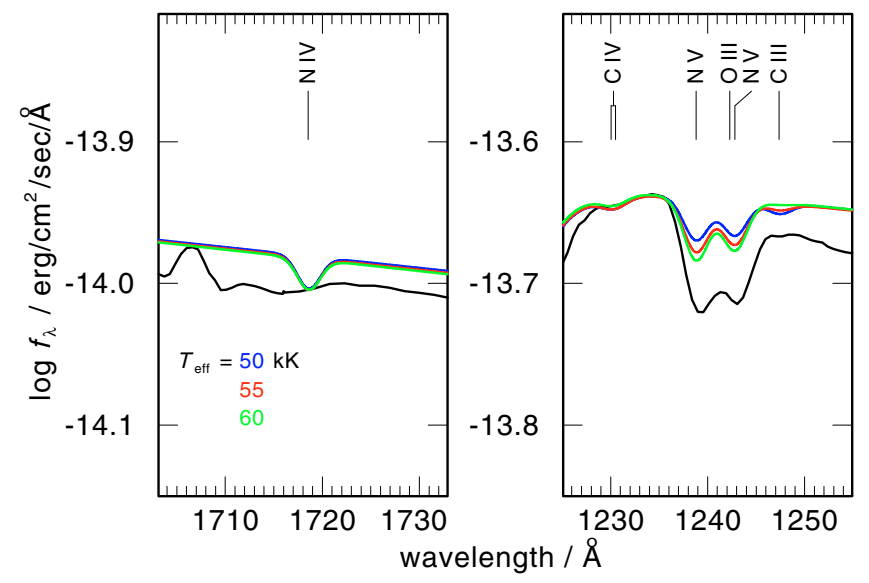

Fig. 11. Stellar model atmosphere fitting of nitrogen lines in the stellar core of TS 01 . Observation is in black.

(black line in the figure), leading to $\exp (-\tau)=0.9$. Therefore, if the measured nebular flux is $F_{\text {neb }} \exp (-\tau)=0.47 \times 2.7 \times 10^{-14}=$ $1.27 \times 10^{-14}$ (last column of Table 3 ), the nebular flux after correction for absorption is $F_{\text {neb }}=1.41 \times 10^{-14}$. The uncertainty is larger here, since the line is weaker. We adopt $30 \%$.

\section{Photoionization modelling}

\subsection{Global strategy}

The chemical composition of TS 01 can only be determined through photoionization modelling, since we have no direct electron temperature diagnostic. With the observational data now at hand, we are able to confine the range of possible abundances much better than in previous studies. In this paper, we try to make the best use of all the observational constraints. The first aspect concerns the morphology. The HST image (see Fig. 1) has an elliptical shape, with two distinct narrow lobes. As the $\mathrm{H} \alpha$ surface brightness distribution shows a hole in the centre, it indicates that those lobes are real (possibly due to jets) and not a thin disc seen edge-on. Because the presence of these lobes can affect the ionization structure of the nebula, we choose to carry out the photoionization modelling with a code that allows us to deal with such geometries: Cloudy_3D (Morisset 2006), based on Ferland's 1D code CLOUDY (Ferland et al. 1998). We use version c07.02.01 of CLOUDY and version 594 of Cloudy_3D. 

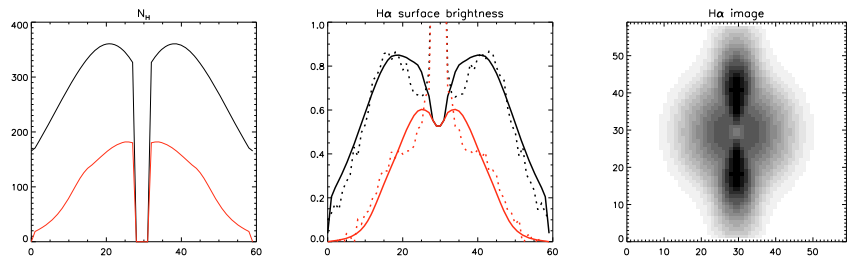

Fig. 12. Density structure of the nebula. Left: the chosen density structure of the model along the polar axis and along an axis perpendicular to it; middle: the resulting $\mathrm{H} \alpha$ surface brightness distribution along the same axes (continuous lines) compared with the observed distribution (dotted lines); right: the theoretical $\mathrm{H} \alpha$ image.

We assume that the nebula is axisymmetric with its large axis in the plane of the sky and that the lobes have a circular cross-section. By trial and error, we choose a density structure to reproduce the observed $\mathrm{H} \alpha$ surface brightness distribution. The chosen density law along the polar axis and along an axis perpendicular to it is shown in the left panel of Fig. 12. The resulting $\mathrm{H} \alpha$ surface brightness distribution along the same axes is shown in the middle panel of this figure (continuous lines) and is compared with the observed distribution ${ }^{5}$ (dotted lines). The right panel shows the theoretical $\mathrm{H} \alpha$ image, which can be compared with the observed image shown in Fig. 1, especially as regards the width of the polar lobes. Note that the density contrast between the lobes and the main body of the nebula is very modest: only a factor of about 2 . The density distribution is parametrized by $n_{0}$, the value of the density at the centre. For each run, we choose $n_{0}$ in a way that, within a circle of a radius of $2.5^{\prime \prime}$, our model returns an $\mathrm{H} \beta$ flux of $2.5 \times 10^{-14} \mathrm{erg} \mathrm{cm}^{-2} \mathrm{~s}^{-1}$, which corresponds to the observed extinction-corrected value from Richer et al. (2002) ${ }^{6}$. The value of $n_{0}$ is thus dependent on the distance $d$ for which the computations are made.

The distance $d$ in turn results from a fitting of the theoretical optical/UV continuum to the observed one (taking into account nebular continuum, aperture effects and reddening).

For the stars, we use model atmospheres computed by T.R. with the Tubingen NLTE model atmosphere package (TMAP). For the cool component, we use models tailored for our object. For the hot component, in absence of sufficient observational constraints, we chose among the complete flux tables for $\mathrm{H}-\mathrm{Ni}$ models with halo composition (May 2001) downloaded from http://astro.uni-tuebingen.de/ rauch. Those models are described in Rauch (2003).

\subsection{The ultraviolet, optical and infrared fluxes on the same scale}

After a model has been run, the extinction-corrected line intensities are computed for each of the observing slits and are compared to the observations. This is the best way to deal with aperture corrections, in particular when combining UV and optical, or IR and optical data. Indeed, such a procedure accounts for the ionization structure of the object under study.

Absolute calibration of spectroscopic observations is notoriously difficult. We intercalibrate the UV/optical data by forcing the measured value of the He II $\lambda 1640 / \mathrm{He}$ II $\lambda 4686$ ratio to the one predicted by our photoionization models in the corresponding slits. The value of $f$ (STIS), representing the factor by which the measured UV fluxes have to be multiplied for the

\footnotetext{
5 For comparison with the model, we have symmetrised the observed nebular surface brightness.

${ }^{6}$ Note that the models are not ionization bounded.
}

He II $\lambda 1640 / \mathrm{He}$ II $\lambda 4686$ ratio to be in agreement with the model, lies between 0.90 and 0.92 in our models. The value of $f$ (STIS) is higher for models with higher electron temperature. To allow an easier comparison between models and observations, we fix the value of $f$ (STIS) to 0.91 .

For Spitzer-IRS observations we multiply the observed fluxes by a factor $f($ IRS) which adjusts the observed values of $[\mathrm{Ne} \mathrm{V}] \lambda 24.3 \mu \mathrm{m} /[\mathrm{Ne} \mathrm{V}] \lambda 3426$ (after reddening correction) to the one predicted by the photoionization model in the corresponding slit. The values of $f$ (IRS) range between 0.87 and 0.95 for the models we considered. It might be judged unreasonable to scale infrared fluxes using the [Ne v] $\lambda 24.3 \mu \mathrm{m} /[\mathrm{Ne} \mathrm{V}] \lambda 3426$ ratio. However, in the electron temperature domain relevant for TS 01 , this ratio does not vary very strongly (from $T_{\mathrm{e}}=20 \mathrm{kK}$ to $40 \mathrm{kK}$, it decreases by only a factor of two). In any case, this is the only option we have to link the Spitzer line fluxes with the optical ones, since our Spitzer data contain no $\mathrm{H}$ or He lines. Of course we bear this difficulty in mind in the discussion. To remove the model dependance of the IR fluxes correction, we fix the value of $f$ (IRS) to 0.91 .

The fact that both $f$ (STIS) and $f$ (IRS) are found very close to unity is remarkable and means that the flux calibration of the STIS and IRS LH spectra of TS 01 is excellent.

\subsection{Judging a model}

To judge a model it is convenient to divide the line ratios to be fitted into different categories:

- ratios of hydrogen lines or of helium lines: they probe the reddening law, the stellar underlying absorption, and the recombination line theory;

- ratios of two different ions of the same element, such as [O IV] $\lambda 25.9 \mu \mathrm{m} /[\mathrm{O}$ III] $\lambda 5007, \mathrm{~N}$ v $\lambda 1240 /[\mathrm{N}$ IV] $\lambda 1486$, [Ne V] $\lambda 3426 /[\mathrm{Ne}$ III] $\lambda 3869$, and [Ne V] $\lambda 3426 /[\mathrm{Ne}$ IV] $\lambda 4720$. They basically test whether the ionization structure is well reproduced by the model. In this category, we add the He II $\lambda 4686 / \mathrm{H} \beta$ line ratio, which is more dependent on the ionization level of the nebula than on the abundance of helium;

- ratios of lines used to determine the chemical composition: [O IV] $\lambda 25.9 \mu \mathrm{m} / \mathrm{H} \beta, \mathrm{C}$ IV $\lambda 1549 / \mathrm{H} \beta, \mathrm{N}$ v $\lambda 1240 / \mathrm{H} \beta$, and $[\mathrm{Ne} \mathrm{V}] \lambda 3426 / \mathrm{H} \beta$. We also consider [O III] $\lambda 5007 / \mathrm{H} \beta$ (although it is redundant with [O IV] $\lambda 25.9 \mu \mathrm{m} / \mathrm{H} \beta$ once the ionization structure is reproduced).

Note that in the case of TS 01 , the only ratio for direct plasma diagnostics (i.e. electron temperature and density) that is available is [Ne V] $\lambda 24.3 \mu \mathrm{m} /[\mathrm{Ne} \mathrm{V}] \lambda 3426$. Unfortunately, the two lines come from measurements in different apertures and with different observing techniques, and as mentioned above, there is a priori some uncertainty in the relative calibration of the two wavelength domains. However, the fact that we find $f($ IRS) close to unity argues that the electron temperature of the $[\mathrm{Ne} \mathrm{V}]$ emitting zone in our models is not far from the true one.

For all the observables considered (usually line ratios), we compute the value of

$\kappa(O)=\left(\log O_{\mathrm{mod}}-\log O_{\mathrm{obs}}\right) / \tau(O)$,

where $O_{\text {mod }}$ is the value returned by the model, $O_{\text {obs }}$ is the observed value, and $\tau(O)$ the accepted tolerance in dex for this observable. For each observable, the value of $\tau(O)$ is chosen a priori considering the observational error bar, including the uncertainty due to reddening, and the expected ability of our model 

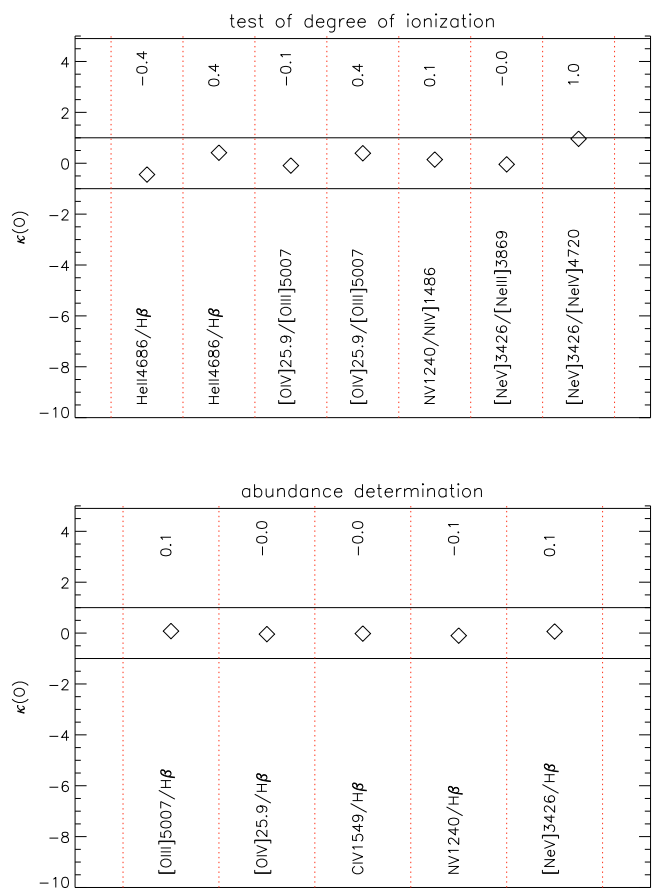

Fig. 13. Graphic chart to compare the reference model with the measured line ratios of TS 01 . The numbers on the top of the panels indicate the values of $\kappa(O)$ for the line ratios listed at the bottom. The line ratios presented in this figure were computed for the following pairs of slits (using the same nomenclature as in Table 5): (0/0), (4/4), (3/0), (3/4), $(1 / 1),(0 / 0)$, and $(0 / 0)$ for the ratios testing the degree of ionization, and $(0 / 0),(3 / 4),(1 / 0),(1 / 0)$, and $(0 / 0)$ for the ratios used to determine the abundances.

to reproduce a given observable. The value of $\tau(O)$ is defined as follows:

$\tau(O)=\log (1+\Delta O / O)$

where $\Delta O$ is the absolute value of the maximum "acceptable" error on the observable. We then judge our models by looking at their outputs presented in graphical form (see an example in Fig. 13). A model is fully satisfying only if each of the values of $\kappa(O)$ is found between -1 and +1 , and if the computed line intensities satisfy the upper limits for undetected lines. Of course, a preliminary condition for a model to be considered is that it returns the correct value of the $\mathrm{H} \beta$ flux, as explained in Sect. 4.1.

\subsection{The reference model}

Here we present our reference model, R. This is the model for which all the values of $\kappa(\mathrm{O})$ are as close as possible to zero, taking the following characteristics for the cool star: $T_{\mathrm{c}}=55 \mathrm{kK}$, $\log g_{\mathrm{c}}=5.1, L_{\mathrm{c}}=1700 L_{\odot}$, and applying the extinction corrections with $R_{V}=3.1$ and $E(B-V)=0.03$ mag as explained in Sect. 3.1. The model contains graphite grains (as expected for a carbon-rich planetary nebula, which is the case of TS 01 as seen below). The grains have a standard size distribution and a total dust-to-gas mass ratio of one tenth of the standard value. A larger abundance of grains would bring the predicted continuum around $24 \mu \mathrm{m}$ into conflict with the observation. Below we explore deviations from the reference model which still account for the observational data.
The reference model has $T_{\mathrm{h}}=170 \mathrm{kK}, \log g_{\mathrm{h}}=6.7$, and a total luminosity $L_{\mathrm{h}}=2564 L_{\odot}^{7} ; n_{0}=181 \mathrm{~cm}^{-3}$ and the following abundances, in units of $12+\log (\mathrm{X} / \mathrm{H}): \mathrm{He}=10.95, \mathrm{C}=7.84$, $\mathrm{N}=7.15, \mathrm{O}=6.82, \mathrm{Ne}=6.83, \mathrm{~S}=5.65, \mathrm{Ar}=4.70$.

The models and observations to which they are compared are presented in Table 5. Column 1 of this table lists the line identifications, Col. 2 characterizes the observation using the following nomenclature: 0 for CFHT 2003, 1 for STIS, 2 for FUSE, 3 for Spitzer, 4 for SDSS, 5 for CFHT 2001. For lines which belong to a wavelength range that was not observed, the number 6 is attributed. Column 3 lists the observed reddening-corrected line intensities (or their upper limits), in units of $\mathrm{H} \beta=100$ in the corresponding apertures. Column 4 lists the acceptable relative error $\Delta \mathrm{O} / \mathrm{O}$ used to compute $\kappa(O)$. In the case of HST, FUSE and Spitzer data, we estimate the value of $\mathrm{H} \beta$ in the relevant aperture, based on our models (since, as explained in Sect. 4, they deliver a smoothed version of the observed surface brightness distribution). The top rows of Col. 5 of the table list the characteristics of the reference model. The predicted line intensities in the relevant aperture are given in the following rows in units of $\mathrm{H} \beta=100$ in the same aperture. For easier analysis, the next rows list a few important line ratios, where the intensity of each line is measured through the aperture corresponding to the observation. In order to shorten the table, we do not list the lines for which the predictions from all our models give values smaller than 0.001 of $\mathrm{H} \beta$ (we note that this is the case for all the recombination lines of elements $\mathrm{C}, \mathrm{N}, \mathrm{O}$ ). We list only the strongest $\mathrm{H}$ and $\mathrm{He}$ lines (we checked that the weaker $\mathrm{H}$ I and He II lines always give $|\kappa(O)|<1-1.5$ in our models, implying that the correction for stellar absorption and reddening is satisfactory).

The graphical representation of the line ratios predicted by model R and used to estimate the chemical composition of TS 01 is shown in Fig. 13. This is the kind of chart that was used in practice when judging the models that were run. A "best model" is one for which all the diamonds fall as close as possible to the ordinate 0 . In any case, an acceptable model should have all line ratios represented by a diamond between the two horizontal lines, which represent a one-sigma deviation from the observed value. In addition, acceptable models should not return line intensities above the upper limits allowed by the observations.

Figure 14 shows the monochromatic images of the reference model in various emission lines. It reveals a few interesting features of the model: some lines, such as [O III] $\lambda 5007$ and [Ne III] $\lambda 3869$ arise mainly in the lobes. Other lines, such as C IV $\lambda 1549, \mathrm{~N}$ V $\lambda 1240$, and [Ne V] $\lambda 3426$ line come from the entire nebula (in agreement with what Fig. 3 suggests for [Ne V] $\lambda 3426$ ), while $\mathrm{O}$ VI $\lambda 1032$ and [Ne VI] $\lambda 7.6 \mu \mathrm{m}$ (the latter not in the observed wavelength range) come from the innermost regions. We can also see that the two C III lines, C III] $\lambda 1909$ and C III $\lambda 977$, although produced by the same ion, come from different regions: C III $\lambda 977$ has an important component coming from the central main body (see Fig. 14), where the very high electron temperature allows for its excitation even if $\mathrm{C}^{++}$is not very abundant there.

Figure 15 compares the observed energy distribution from TS01 with the computed one. The top panel shows the reddening-corrected flux computed for model $\mathrm{R}$, in erg $\mathrm{cm}^{-2} \mathrm{~s}^{-1} \AA^{-1}$, in the wavelength range of $900 \AA-40 \mu \mathrm{m}$. The observations are superimposed in various colours, as indicated in the caption. One can see that the model reproduces

7 The mass of the hot stellar component in the models depends on the value assumed for the gravity, which is not well constrained. For the photoionization modelling, what really matters is $T_{\mathrm{h}}$ and $L_{\mathrm{h}}$. 

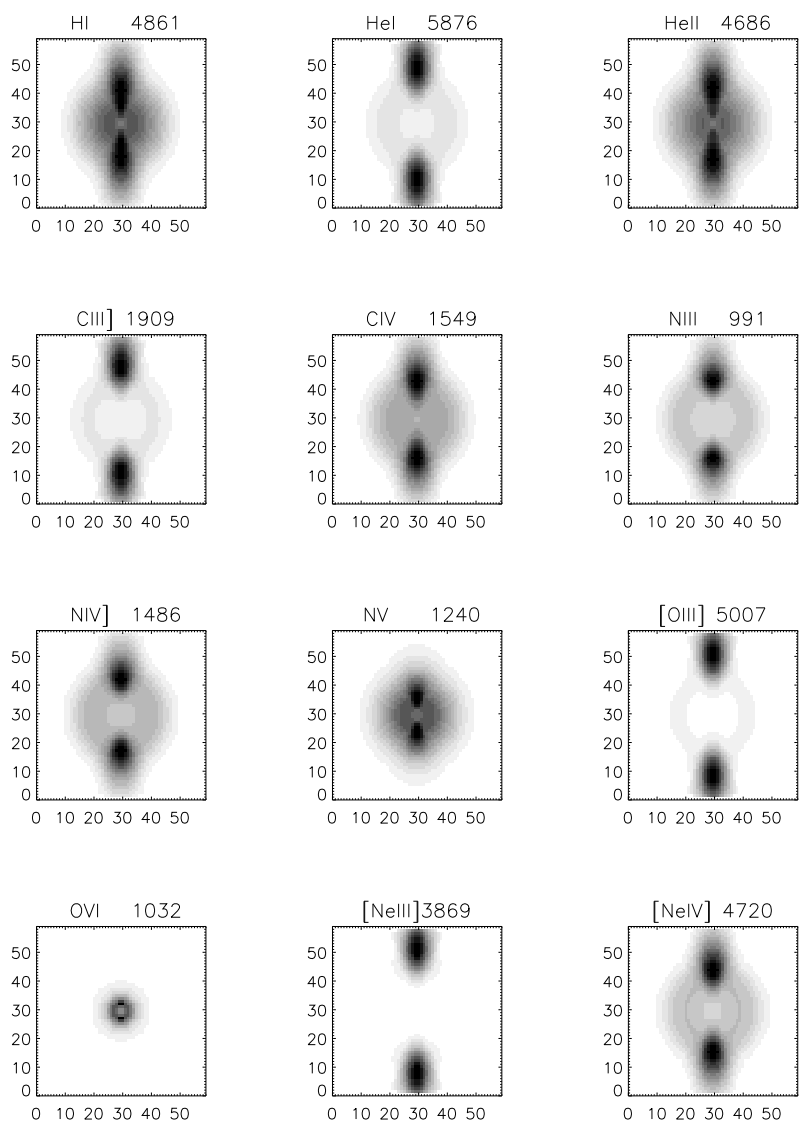

Fig. 14. Monochromatic images of the reference model in various lines (the values of the wavelengths are in $\AA$ if they are larger than 900, and in $\mu$ m otherwise). The $x$ and $y$ values are the coordinates in pixel units of the models.

the observed spectral energy distribution quite well (except for the IRS SH observations which could not be corrected for sky emission, as explained in Sect. 2.4). The bottom panel shows the energy distribution in the soft X-ray range: the blue curve is the hot star, the green triangles are the XMM observations. The flux from the star has been corrected for the nebular absorption (computed by CLOUDY) and for the interstellar absorption, taking a hydrogen column density of $1.6 \times 10^{20} \mathrm{~cm}^{-2}$. For each computed model we checked that the ionizing flux does not violate the observed stellar emission up to $200 \mathrm{eV}$. At higher energies, the observed emission may have another origin than the stars we consider, but it does not affect our model fitting, since we have no relevant observational constraints (the ion with the highest ionization potential observed is $\mathrm{Ne}^{4+}$, which has an ionization potential of $97.1 \mathrm{eV}$ ).

Figure 16 compares the energy distributions of the two stars considered in the modelling: the "cool" star is in red, the "hot" one is in blue. The sum of the two is in black. As mentioned in Sect. 3.3, the cool star dominates in the optical range (a few eV) and until about $20 \mathrm{eV}$, but it is the hot star which provides the photons with energies above $40-50 \mathrm{eV}$. Consequently it is the cool star which provides most of the $\mathrm{H}$ ionizing photons, but it is the hot star which provides the photons responsible for the presence of the He II, N V, [O IV], [Ne IV], [Ne V] and [Ar V] lines. This is a very uncommon situation, perhaps a unique case among planetary nebulae: TS 01 has two ionizing stars! This explains why our previous attempts to model the object were facing the difficulty that the nebula needed plenty of photons of energies
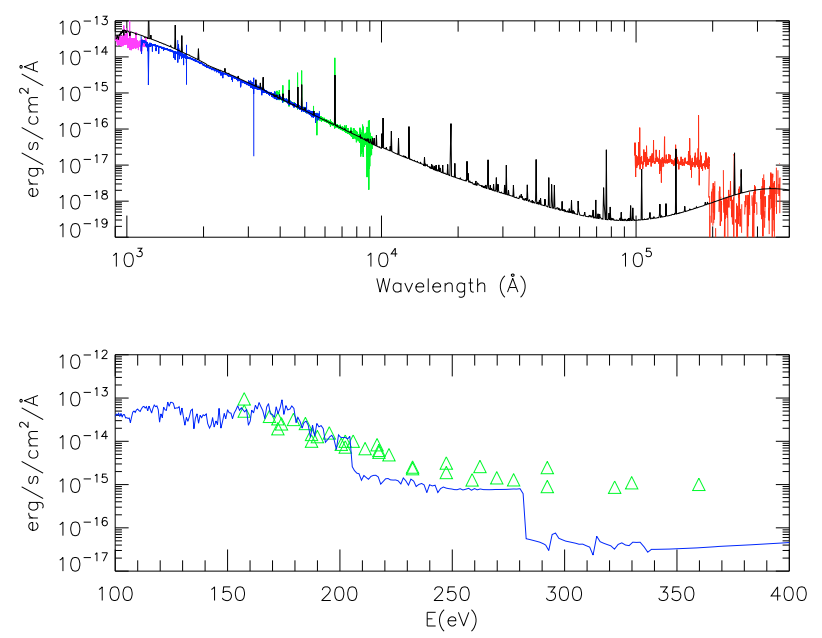

Fig. 15. Comparison of the reddened spectrum of the reference model with observations. Top: from the UV to the IR. The reddened model is in black. The colour code for the observations is as follows. Magenta: FUSE; blue: HST; green: SDSS ; red: Spitzer (the SH observations could not be sky-corrected). Bottom: X-ray domain. The model (with extinction applied) is in blue. The XMM observations are represented by triangles.

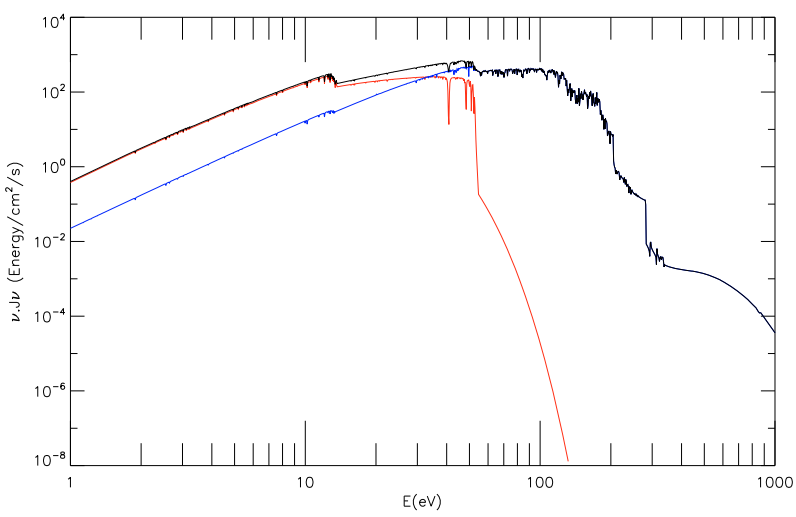

Fig. 16. Spectral energy distribution (normalized to arbitrary units) of the radiation from the ionizing stars in the reference model. Red: the "cold" star; blue: the "hot" star; black: the sum of the two.

above $54.4 \mathrm{eV}$, while the Balmer absorption lines in the stellar continuum indicated a moderate temperature.

From Figs. 13, 15, and Table 5 one can see that our reference model fits all the observational constraints very well. The only exception is that of C III] $\lambda 1909$, whose intensity is slightly above the upper limit we gave to the STIS observation. However, we consider this result to be still acceptable, since upper limit fluxes for unobserved lines are difficult to estimate accurately. The abundances of $\mathrm{C}, \mathrm{N}, \mathrm{O}$, and $\mathrm{Ne}$ in the reference model are, respectively, 1/3.5, 1/4.2, 1/70, and 1/11, and, for $\mathrm{S}$ and $\mathrm{Ar}<$ $1 / 30$ of the solar values given by Asplund et al. (2005).

\section{The chemical composition of TS 01}

\subsection{Range of abundances for the reference model}

We now investigate the error bars on abundances that are due only to the uncertainties in the observed line intensities. Since $\mathrm{C}, \mathrm{N}, \mathrm{O}$, and $\mathrm{Ne}$ contribute very little to the energy budget, it is straightforward to estimate from model $\mathrm{R}$ the minimum and maximum abundances corresponding to the minimum and maximum values of [O IV] $\lambda 25.9 \mu \mathrm{m} / \mathrm{H} \beta, \mathrm{C}$ IV $\lambda 1549 / \mathrm{H} \beta$, 
A\&A 511, A44 (2010)

Table 5. Photoionization models versus observations. Line intensities are in units of $\mathrm{H} \beta=100$ in the corresponding aperture.

\begin{tabular}{|c|c|c|c|c|c|c|c|c|}
\hline (1) & $(2)^{a}$ & (3) & (4) & $\begin{array}{r}\text { (5) } \\
\mathrm{R} \\
\end{array}$ & $\begin{array}{l}(6) \\
\mathrm{Mi} \\
\end{array}$ & $\begin{array}{l}(7) \\
\mathrm{Ma} \\
\end{array}$ & $\begin{array}{r}(8) \\
\mathrm{HeMi} \\
\end{array}$ & $\begin{array}{r}(9) \\
\text { HeMa }\end{array}$ \\
\hline & & $T_{\mathrm{h}}$ & $10^{3} \mathrm{~K}$ & 170 & 170 & 170 & 170 & 170 \\
\hline & & $L_{\mathrm{h}}$ & {$\left[L_{\odot}\right]$} & 2564 & 1618 & 4064 & 1618 & 4064 \\
\hline & & $\log g_{\mathrm{h}}$ & & 6.7 & 6.7 & 6.7 & 6.7 & 6.7 \\
\hline & & $n_{0}$ & $\mathrm{~cm}^{-3}$ & 181 & 181 & 181 & 181 & 181 \\
\hline \multicolumn{9}{|l|}{$\operatorname{Sun}^{b}$} \\
\hline 10.93 & & $\mathrm{He}^{c}$ & & 10.95 & 10.95 & 10.95 & 10.98 & 10.91 \\
\hline 8.39 & & $\mathrm{C}^{c}$ & & 7.84 & 7.64 & 8.05 & 7.64 & 8.05 \\
\hline 7.78 & & $\mathrm{~N}^{c}$ & & 7.15 & 7 & 7.32 & 7 & 7.32 \\
\hline 8.66 & & $\mathrm{O}^{c}$ & & 6.82 & 6.63 & 7.13 & 6.63 & 7.13 \\
\hline 7.85 & & $\mathrm{Ne}^{c}$ & & 6.83 & 6.76 & 6.9 & 6.76 & 6.9 \\
\hline 7.14 & & $\mathrm{~S}^{c}$ & & 5.65 & 5.5 & 5.83 & 5.5 & 5.83 \\
\hline \multirow[t]{7}{*}{6.18} & & $\mathrm{Ar}^{c}$ & & 4.7 & 4.5 & 4.92 & 4.5 & 4.92 \\
\hline & & $d$ & {$[\mathrm{kpc}]$} & 22.5 & 22.3 & 22.9 & 22.3 & 22.9 \\
\hline & & $F_{\beta}{ }^{d}$ & & 2.55 & 2.52 & 2.56 & 2.54 & 2.54 \\
\hline & & $M(\mathrm{H})$ & {$\left[M_{\odot}\right]$} & 0 & 0.14 & 0.15 & 0.14 & 0.15 \\
\hline & & $f(\mathrm{STIS})$ & & 0.91 & 0.91 & 0.91 & 0.92 & 0.91 \\
\hline & & $f($ IRS $)$ & & 0.91 & 0.88 & 0.94 & 0.87 & 0.95 \\
\hline & & & $\Delta \mathrm{O} / \mathrm{O}$ & & & & & \\
\hline $\mathrm{H} 8$ & 0 & 9.51 & 0.15 & 9.01 & 9.02 & 9.02 & 8.96 & 9.09 \\
\hline $\mathrm{H} 8$ & 4 & 9.29 & 0.15 & 9.15 & 9.19 & 9.14 & 9.12 & 9.21 \\
\hline $\mathrm{H} 7$ & 0 & 12.99 & 0.1 & 14.18 & 14.22 & 14.18 & 14.12 & 14.28 \\
\hline $\mathrm{H} 7$ & 4 & 13.6 & 0.1 & 14.39 & 14.46 & 14.36 & 14.36 & 14.47 \\
\hline H6 & 0 & 25.66 & 0.07 & 23.64 & 23.70 & 23.64 & 23.54 & 23.81 \\
\hline H6 & 4 & 25.71 & 0.07 & 23.87 & 23.96 & 23.85 & 23.80 & 24.03 \\
\hline $\mathrm{H} \gamma$ & 0 & 47.84 & 0.05 & 47.40 & 47.47 & 47.34 & 47.41 & 47.41 \\
\hline $\mathrm{H} \gamma$ & 4 & 46.73 & 0.05 & 47.64 & 47.71 & 47.59 & 47.65 & 47.66 \\
\hline $\mathrm{H} \alpha$ & 5 & 251.41 & 0.04 & 282.06 & 282.48 & 281.30 & 283.21 & 280.50 \\
\hline $\mathrm{H} \alpha$ & 4 & 311.03 & 0.04 & 279.89 & 280.14 & 279.23 & 280.85 & 278.43 \\
\hline HeI5876 & 5 & $<0.19$ & & 0.16 & 0.25 & 0.11 & 0.27 & 0.10 \\
\hline HeI7065 & 4 & $<1.44$ & & 0.04 & 0.06 & 0.03 & 0.06 & 0.02 \\
\hline HeII1640 & 1 & 609.18 & 0.1 & 594.53 & 572.27 & 623.3 & 607.55 & 575.72 \\
\hline HeII4686 & 0 & 77.88 & 0.04 & 76.53 & 73.85 & 79.67 & 78.35 & 73.60 \\
\hline HeII4686 & 4 & 74.93 & 0.04 & 76.16 & 74.00 & 78.91 & 78.52 & 72.93 \\
\hline HeII5412 & 4 & 5.43 & 0.1 & 6.19 & 6.02 & 6.38 & 6.40 & 5.88 \\
\hline CIII]1909 & 1 & $<61.44$ & & 73.41 & 89.01 & 59.96 & 93.05 & 56.73 \\
\hline CIII977 & 2 & $<430.3$ & & 104.58 & 112.54 & 96.78 & 117.74 & 92.22 \\
\hline CIV1549 & 1 & 1054.84 & 0.2 & 1050.89 & 890.87 & 1246.07 & 916.75 & 1200.76 \\
\hline CIV4659 & 0 & $<0.38$ & & 0.14 & 0.08 & 0.26 & 0.08 & 0.26 \\
\hline [NIII] 57.2 & 6 & & & 1.23 & 1.54 & 0.96 & 1.56 & 0.93 \\
\hline NIII] 1750 & 1 & $<60.84$ & & 3.00 & 4.29 & 1.99 & 4.49 & 1.87 \\
\hline NIII991 & 2 & $<423.77$ & & 7.54 & 9.52 & 5.91 & 9.81 & 5.69 \\
\hline NIV]1486 & 1 & 73.05 & 0.5 & 65.89 & 67.40 & 61.95 & 69.93 & 58.82 \\
\hline NV1240 & 1 & 339.3 & 0.3 & 330.38 & 253.7 & 425.38 & 259.25 & 410.86 \\
\hline [OIII]4363 & 0 & $<0.42$ & & 0.11 & 0.14 & 0.12 & 0.15 & 0.11 \\
\hline [OIII]5007 & 0 & 2.52 & 0.3 & 2.57 & 3.24 & 2.58 & 3.35 & 2.49 \\
\hline OIV]1402 & 1 & $<36.97$ & & 7.68 & 6.88 & 10.60 & 7.15 & 10.05 \\
\hline [OIV]25.9 & 3 & 13.72 & 0.3 & 13.57 & 11.20 & 20.35 & 11.24 & 20.13 \\
\hline OVI1032 & 2 & $<407.42$ & & 50.48 & 30.45 & 105.06 & 30.65 & 102.45 \\
\hline [NeIII]3869 & 0 & 0.79 & 0.5 & 0.82 & 1.45 & 0.44 & 1.50 & 0.43 \\
\hline$[\mathrm{NeIII}] 15.5$ & 3 & $<7.22$ & & 0.21 & 0.37 & 0.11 & 0.38 & 0.11 \\
\hline$[\mathrm{NeIII}] 36.0$ & 3 & $<28.88$ & & 0.02 & 0.03 & 0.01 & 0.03 & 0.01 \\
\hline [NeIV]2424 & 6 & & & 25.20 & 29.46 & 20.51 & 30.10 & 19.93 \\
\hline$[\mathrm{NeV}] 3426$ & 0 & 57.67 & 0.2 & 58.32 & 48.63 & 66.92 & 49.19 & 65.84 \\
\hline$[\mathrm{NeV}] 14.3$ & 3 & 29.62 & 0.3 & 43.25 & 35.05 & 50.69 & 34.84 & 50.91 \\
\hline$[\mathrm{NeV}] 24.3$ & 3 & 54.17 & 0.2 & 54.44 & 44.16 & 63.76 & 43.90 & 64.03 \\
\hline$[\mathrm{NeVI}] 7.6$ & 6 & & & 27.47 & 15.98 & 45.07 & 15.84 & 45.48 \\
\hline [ArIV]4711 & 0 & $<0.15$ & & 0.15 & 0.15 & 0.14 & 0.16 & 0.13 \\
\hline [ArIV] 4740 & 0 & $<0.15$ & & 0.12 & 0.12 & 0.11 & 0.13 & 0.10 \\
\hline$[\mathrm{ArV}] 7005$ & 4 & $<1.44$ & & 0.27 & 0.23 & 0.31 & 0.23 & 0.30 \\
\hline
\end{tabular}


Table 5. continued.

\begin{tabular}{|c|c|c|c|c|c|c|c|}
\hline (1) & $(2)^{a}$ & (3) (4) & $\begin{array}{r}\text { (5) } \\
\mathrm{R}\end{array}$ & $\begin{array}{l}\text { (6) } \\
\mathrm{Mi}\end{array}$ & $\begin{array}{l}\text { (7) } \\
\mathrm{Ma}\end{array}$ & $\begin{array}{r}(8) \\
\mathrm{HeMi}\end{array}$ & $\begin{array}{r}(9) \\
\mathrm{HeMa}\end{array}$ \\
\hline$[\mathrm{ArV}] 8.0$ & 6 & & 0.29 & 0.22 & 0.37 & 0.22 & 0.36 \\
\hline [ArV]13.1 & 3 & $<7.23$ & 0.45 & 0.33 & 0.57 & 0.33 & 0.57 \\
\hline [SIII]9069 & 4 & $<1.41$ & 0.24 & 0.31 & 0.17 & 0.32 & 0.16 \\
\hline [SIII] 18.7 & 3 & $<7.22$ & 0.21 & 0.25 & 0.17 & 0.25 & 0.16 \\
\hline$[\mathrm{SIII}] 33.5$ & 3 & $<36.1$ & 0.34 & 0.41 & 0.28 & 0.42 & 0.27 \\
\hline [SIV]10.5 & 3 & $<7.23$ & 6.65 & 6.29 & 6.88 & 6.35 & 6.74 \\
\hline NV1240/ NIV]1486 & $1 / 1$ & 4.6450 .7 & 5.01 & 3.76 & 6.86 & 3.71 & 6.98 \\
\hline [OIV]25.9/[OIII]5007 & $3 / 0$ & 0.1390 .4 & 0.13 & 0.087 & 0.20 & 0.085 & 0.21 \\
\hline$[\mathrm{OIV}] 25.9 /[\mathrm{OIII}] 5007$ & $3 / 4$ & 0.1940 .4 & 0.22 & 0.136 & 0.35 & 0.134 & 0.36 \\
\hline$[\mathrm{NeV}] 3426 /[\mathrm{NeIII}] 3869$ & $0 / 0$ & 73.0210 .7 & 71.33 & 33.47 & 151.80 & 32.84 & 154.26 \\
\hline$[\mathrm{NeV}] 3426 /[\mathrm{NeIV}] 4720$ & $0 / 0$ & 99.0390 .3 & 127.42 & 89.66 & 181.34 & 87.90 & 185.72 \\
\hline$[\mathrm{NeV}] 24.3 /[\mathrm{NeV}] 3426$ & $3 / 0$ & 0.0240 .3 & 0.024 & 0.023 & 0.024 & 0.020 & 0.025 \\
\hline$[\mathrm{NeV}] 24.3 /[\mathrm{NeV}] 14.3$ & $3 / 3$ & 1.8280 .3 & 1.26 & 1.26 & 1.26 & 1.26 & 1.26 \\
\hline
\end{tabular}

Notes. ${ }^{(a)}$ Labels for the observing slits: 0: CFHT 2003; 1: STIS; 2: FUSE; 3: Spitzer; 4: SDSS; 5 : CFHT 2001; ${ }^{(b)}$ Asplund, Grevesse \& Sauval (2005); ${ }^{(c)}$ abundances in units of $12+\log \mathrm{X} / \mathrm{H} ;{ }^{(d)}$ in units of $10^{-14} \mathrm{erg} \mathrm{cm}^{-2} \mathrm{~s}^{-1}$.

$\mathrm{N} \vee \lambda 1240 / \mathrm{H} \beta$, and $[\mathrm{Ne} \mathrm{V}] \lambda 3426 / \mathrm{H} \beta$ without changing the ionization structure of the nebula. However, one has also to consider the error bars on line ratios that constrain the ionization structure: [O IV] $\lambda 25.9 \mu \mathrm{m} /[\mathrm{O}$ III] $\lambda 5007, \mathrm{~N}$ v $\lambda 1240 /[\mathrm{N}$ IV] $\lambda 1486,[\mathrm{Ne} \mathrm{V}] \lambda 3426 /[\mathrm{Ne}$ III] $\lambda 3869$, and [Ne V] $\lambda 3426 /[\mathrm{Ne}$ IV] 24720. The minimum values of the $\mathrm{C}, \mathrm{N}, \mathrm{O}$, and $\mathrm{Ne}$ abundances in TS 01 are obtained by a model with the lowest ionization compatible with the observations and the lower limits of [O IV] $\lambda 25.9 \mu \mathrm{m} / \mathrm{H} \beta, \mathrm{C}$ IV $\lambda 1549 / \mathrm{H} \beta, \mathrm{N}$ V $\lambda 1240 / \mathrm{H} \beta$, and [Ne v] $\lambda 3426 / \mathrm{H} \beta$. Such a model, Mi, is reported in Col. 6 of Table 5 . It is derived from the reference model $\mathrm{R}$ by lowering the values of $L_{\mathrm{h}}$, and decreasing the values of the $\mathrm{C}, \mathrm{N}, \mathrm{O}$, and $\mathrm{Ne}$ abundances. With similar considerations, one can construct a model $\mathrm{Ma}$, which will give the maximum $\mathrm{C}, \mathrm{N}, \mathrm{O}$, and Ne abundances. This model, with a higher $L_{\mathrm{h}}$ and same $T_{\mathrm{h}}$ is listed in Col. 7 of Table 5 .

The resulting limits on the abundances of $\mathrm{C}, \mathrm{N}, \mathrm{O}$, and $\mathrm{Ne}$ in the gaseous phase ${ }^{8}$ of TS 01 are thus:

$7.64<12+\log \mathrm{C} / \mathrm{H}<8.05$

$7.00<12+\log \mathrm{N} / \mathrm{H}<7.32$

$6.63<12+\log \mathrm{O} / \mathrm{H}<7.13$

$6.76<12+\log \mathrm{Ne} / \mathrm{H}<6.90$.

The limits on the $\mathrm{C} / \mathrm{O}, \mathrm{N} / \mathrm{O}$ and $\mathrm{Ne} / \mathrm{O}$ ratios are obtained by considering tailored models which reproduce the extreme values of the observed intensities of the $\mathrm{C}, \mathrm{N}$ and $\mathrm{Ne}$ lines. They are:

$0.83<\log \mathrm{C} / \mathrm{O}<1.21$

$0.12<\log \mathrm{N} / \mathrm{O}<0.54$

$-0.16<\log \mathrm{Ne} / \mathrm{O}<0.17$.

To derive the lower limit on $\mathrm{He} / \mathrm{H}$, one must consider the model with the lowest ionization compatible with the observed [O IV] $\lambda 25.9 \mu \mathrm{m} /[\mathrm{O}$ III] $\lambda 5007, \mathrm{~N}$ V $\lambda 1240 /[\mathrm{N}$ IV] $\lambda 1486$, [Ne V] $\lambda 3426 /[\mathrm{Ne} \mathrm{III]} \lambda 3869$, and [Ne V] $\lambda 3426 /[\mathrm{Ne} \mathrm{IV]} \lambda 4720$,

8 The contribution of grains to the abundance of carbon is discussed in Sect. 5.2.2. and the lowest He II $\lambda 4686 / \mathrm{H} \beta$. The upper limit on $\mathrm{He} / \mathrm{H}$ is obtained with similar arguments. The corresponding models HeMi and HeMa, respectively, are listed in Cols. 8 and 9 of Table 5. The resulting limits for $\mathrm{He} / \mathrm{H}$ obtained in this way are 0.095 and 0.081 . In other words, the precision on the $\mathrm{He} / \mathrm{H}$ abundance is poor, despite the fact that we have been fitting the He II $\lambda 4686 / \mathrm{H} \beta$ ratio within $4 \%$ (the formal uncertainty in this ratio is $2 \%$ for the 2003 CFHT data, but a comparison with SDSS data led us to adopt a higher value for the tolerance). With a more accurate upper limit on $\mathrm{He} \mathrm{I} \lambda 5876 / \mathrm{H} \beta$ we could reduce the error bar on the helium abundance (and actually, model HeMi slightly violates the present upper limit on $\mathrm{He} \mathrm{I} \lambda(5876 / \mathrm{H} \beta)$. But the uncertainty in $\mathrm{He} / \mathrm{H}$ will remain larger than the uncertainty in the $\mathrm{He}$ II $\lambda 4686 / \mathrm{H} \beta$ ratio mainly because the electron temperature gradient in this nebula is steep and the ratio of emissivities of He II $\lambda 4686$ and $\mathrm{H} \beta$ slightly varies with temperature.

\subsection{Additional sources of abundance uncertainties}

In this section, we discuss how reasonable variations of the parameters that were so far fixed in the modelling procedure affect the derived abundances. We also discuss some more general problems that may have an influence on the estimated chemical composition of TS 01 . To save space, the models that were constructed to discuss these additional uncertainties are not listed in the paper. We will only mention their impact on the derived abundances. Note that all of those additional models have the required angular size and total $\mathrm{H} \beta$ flux, and their abundances have been chosen to fit the observed emission line ratios.

\subsubsection{The effect of changing the description of the stars}

So far, we have kept the parameters of the cool star fixed. Even if they are rather well determined, as explained in Sect. 3.3 and Tovmassian et al. (in prep.), it is important to see the effect that a change in those parameters implies on the derived chemical composition of the nebula. It turns out that an increase of $5 \mathrm{kK}$ in $T_{\mathrm{c}}$ induces a decrease in the $\mathrm{C}, \mathrm{N}, \mathrm{O}$ and $\mathrm{Ne}$ abundances by $0.05-0.08$ dex. A change in $g_{\mathrm{c}}$ by 0.1 dex leaves the abundances of the fitted nebular model unchanged. 
We then explored the effects of changing the parameters of the hot star. By increasing the temperature of the hot star by $10 \mathrm{kK}$, (implying a slight decrease of its luminosity to fit the observations) one decreases the $\mathrm{C}, \mathrm{N}, \mathrm{O}, \mathrm{Ne}$ abundances of the fitted nebular model by about 0.2 dex.

We also explored the effect of changing the model atmosphere of the hot star. One extreme case is to consider a model atmosphere composed only of $\mathrm{H}$ and $\mathrm{He}$, instead of the Galactic halo chemical composition. Consequently, there are no absorption edges in the atmosphere above $54 \mathrm{eV}$. The model which fits the observations presents an intense [Ne VI] $\lambda 7.6 \mu \mathrm{m}$ emission (unfortunately outside the wavelength range of our IRS observations of TS 01). Its Ne abundance is higher by 0.15 dex than that of the reference model, while the abundances of the remaining elements are almost unchanged.

\subsubsection{Dust issues}

Concerning the extinction and reddening issues, a change of $E(B-V)$ and $R_{V}$ within limits compatible with the observed Balmer decrement and the observed stellar energy distribution does not alter the abundances derived for TS 01 significantly.

Our reference model has a dust-to-gas mass ratio of $10^{-1}$ times the canonical value, with the canonical grain size distribution as stated in Sect. 4.4. The chemical composition of the grains - pure graphite - is dictated by the fact that the object is undoubtedly carbon-rich, as seen in Sects. 3.3 and 5.1. The total abundance of grains in the reference model is chosen in a way that the predicted infrared flux which arises from the heated grains does not exceed the observed IRS LH flux and that it produces no significant dip at $2200 \AA$, since this is not observed. The total amount of carbon locked in grains in the reference model is 0.4 times that of the abundance of carbon in the gas phase. This means that the total abundance of carbon in the nebula (gas plus grains) is larger by about 0.15 dex than given in Sect. 5.1.

\subsubsection{The role of morphology}

While constructing our reference model (and all the models described before), we have chosen a geometry that reproduces the observed $\mathrm{H} \alpha$ surface brightness, including the lobes. It is interesting to experiment with a simpler model without any lobes, in which the averaged surface brightness is the same as in the reference model. The abundances in such a model differ insignificantly from those of the reference model. We have to confess that we were somewhat surprised by this result, since as shown in Fig. 14 the emission in such lines as [O III] $\lambda 5007$ or [Ne III] $\lambda 3869$ traces the lobes very distinctly. On the other hand, one has to remember that the density contrast between the lobes and the ambient medium is only a factor of two, as seen in Fig. 12.

\subsection{Caveats}

\subsubsection{The problem of $\mathrm{H} \alpha$}

One of the intriguing problems in the observations of TS 01 is the behaviour of the $\mathrm{H} \alpha$ line. As seen in Table 2, the observed $\mathrm{H} \alpha / \mathrm{H} \beta$ ratio varies among data taken during different runs and at different telescopes. Since the ratios of all the remaining hydrogen lines look normal within the error bars, we are inclined to think that this $\mathrm{H} \alpha$ problem has no influence on the derived chemical composition. Nevertheless, we feel it important to try to understand the reason for the observed values of $\mathrm{H} \alpha / \mathrm{H} \beta$.
In the present study, we have done the computations with the full treatment of hydrogen as offered by CLOUDY (this, and not case $\mathrm{B}$, is actually the default option in CLOUDY). Under the physical conditions in this nebula, one indeed does not expect the Balmer lines to be emitted under case B, not even with the added effect of collisional excitation. The ionization parameter of the emitting regions is high and the nebula is optically thin, which renders it a good candidate for case $\mathrm{C}$ as described by Baker \& Menzel (1938) and reconsidered by Ferland (1999). In such a case, absorption of Lyman photons from the star contributes to the emission of the Balmer lines, and the Balmer decrement depends on the number of respective Lyman line photons in the star. However, we are far from reproducing the $\mathrm{H} \alpha / \mathrm{H} \beta$ ratios observed in the various slits. Of course, the computed Balmer decrement strongly depends on the fluxes at the wavelengths of the H Lyman lines in the model atmosphere used. But the differences in the $\mathrm{H} \alpha / \mathrm{H} \beta$ ratios in the different observing runs make it doubtful that simple stellar fluorescence can explain the observations.

The reference model predicts a ratio of about 2.81 . The differences in the observed $\mathrm{H} \alpha / \mathrm{H} \beta$ ratios cannot have a nebular origin since the associated time scales are far too long.

Water vapour absorption near $\mathrm{H} \alpha$ is far too weak to explain the variations 9 . Now that the nature of the binary central star is better known, we can also discard the possibility that much of the $\mathrm{H} \alpha$ emission comes from an accretion disc. Active mass transfer in the system has ceased and, even if there is a stellar wind or weak remnant of an accretion disc around the hot component, it cannot have a big influence on emission lines, since we detect fairly symmetric underlying absorption lines from the cool component at all orbital phases. These symmetric lines also imply that extra emission from the irradiated face of the cool component does not contribute any significant $\mathrm{H} \alpha$ emission.

The remaining option is atmospheric refraction (Filippenko 1982), since the slit was not oriented at the parallactic angle for many (though not all) of the spectra with $\mathrm{H} \alpha / \mathrm{H} \beta$ ratios differing significantly from 2.81 (rather, usually east-west). What is odd a priori, if atmospheric refraction is responsible, is that the lines from $\mathrm{H} \delta$ to $\mathrm{H} \beta$ are observed with constant intensity ratios. Simulations in which we convolve the quantum efficiency of the slit camera used at SPM ${ }^{10}$ with the object's very blue spectrum indicate that the effective wavelength is between $4000 \AA$ and $4500 \AA$. Thus, the effective wavelength, which is what is used to centre the object in the slit, is between the blue lines, so atmospheric refraction has very little effect upon them. As a result, $\mathrm{H} \alpha$ should be the only optical line that may be significantly affected by atmospheric refraction. Also, compared to the usual assumptions, the wavelength baseline over which atmospheric refraction operates is unusually large in this case, of the order of $2000 \AA$ or more. Tests using the SPM4 dataset (Richer et al. 2002), in which this issue can be studied in greatest detail, clearly implicate the effect of atmospheric refraction since the spectral shape of the central star's continuum varies as a function of the difference between the slit position angle and the parallactic angle. Therefore we are inclined to attribute the variations observed in the $\mathrm{H} \alpha / \mathrm{H} \beta$ ratio to atmospheric refraction.

\footnotetext{
9 http://www.astrossp.unam.mx/sitio/ abs_telurica_english.htm

10 http://wWW . astrossp. unam.mx//Instruments/bchivens/ camrend/manual-english.pdf
} 


\subsubsection{Atomic data}

As noted by Péquignot \& Tsamis (2005), the atomic data on which photoionization models are built are not of perfect accuracy. All the models we have computed rely on CLOUDY c07.02.01. It is not excluded that future advances in atomic physics, especially in the calculation of recombination coefficients for highly ionized species, might affect the computed ionization structure. However, the fact that we now have observational data (or stringent upper limits) on several ions of each of the elements $\mathrm{C}, \mathrm{N}, \mathrm{O}$ and $\mathrm{Ne}$ in TS 01 makes us confident in the robustness of the chemical composition that we have derived. The relatively large error bars we obtain on the abundances (principally due to the lack of a direct measure of the electron temperature in the nebula) imply that the uncertainties in atomic data, including the collision strengths of the lines used for the diagnostics, should be negligible in the total error budget.

\subsubsection{Dynamical effects}

Schönberner et al. (2005) have drawn attention to the possible importance of dynamical effects in the thermal balance of nebulae. They make the point that the role of dynamical expansion in the cooling budget increases as the metallicity decreases. We have therefore included the effect of expansion cooling in CLOUDY by introducing a wind cooling contribution in the routine ' 'cool\_eval.cpp' ':

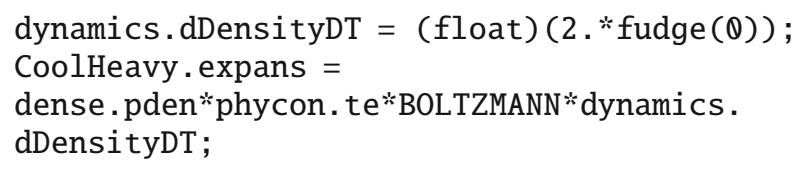

with the user defined parameter "fudge" related to the expansion velocity and the outer radius of the nebula by fudge" $=v_{\exp } / R_{\text {out }}$.

All the models presented above have been computed with an expansion velocity of $30 \mathrm{~km} \mathrm{~s}^{-1}$, corresponding to the observed value (see Sect. 3.2). We have tried other values for $v_{\exp }$ in the equation above, but noted no significant changes in the output between 0 and $200 \mathrm{~km} \mathrm{~s}^{-1}$, the extreme values we tried. This result is at variance with the finding by Schönberner et al. (2005) that expansion cooling significantly reduces the temperature with respect to a fully static model of the same density structure.

In our models, the dominant cooling process is collisional excitation of $\mathrm{H} \operatorname{Ly} \alpha$, and, at the ionization level predicted by the model, it is clear that expansion cooling must be negligible, unless the velocity of the jet is of the order of $1000 \mathrm{~km} \mathrm{~s}^{-1}$.

Could it be that the lower temperature found by Schönberner et al. (2005) in fully dynamical models with respect to hydrostatic ones, which they attribute to expansion cooling, is actually the result of some other process? The only idea that comes to mind is a departure from ionization equilibrium. For an average temperature of $30 \mathrm{kK}$ and an average density of about $200 \mathrm{~cm}^{-3}$, the recombination time for hydrogen is about $10^{3} \mathrm{yr}$. From the apparent size, expansion velocity and distance to TS01, one can estimate an expansion time of $\sim 7 \times 10^{3} \mathrm{yr}$. Therefore, the nebula should not be far from ionization equilibrium. On the other hand, the dynamical model shown in Schönberner et al. (2005) was for a $0.595 M_{\odot}$ star with an effective temperature of $100 \mathrm{kK}$, corresponding to an evolution time of $\sim 5 \times 10^{3} \mathrm{yr}$. The average density of the nebula in their simulation is then about $100 \mathrm{~cm}^{-3}$. In such a situation, the nebula is farther from ionization equilibrium. Since their star is in a phase where the number of ionizing
Table 6. Nebular abundances of TS 01, in various units.

\begin{tabular}{lrlcc}
\hline \hline & $12+\log \mathrm{X} / \mathrm{H}$ & Uncertainty & $\mathrm{X} / \mathrm{H}$ & Mass fraction \\
\hline $\mathrm{He}$ & 10.95 & \pm 0.04 & $8.91 \times 10^{-2}$ & $2.63 \times 10^{-1}$ \\
$\mathrm{C}$ & 7.84 & \pm 0.30 & $6.92 \times 10^{-5}$ & $6.11 \times 10^{-4}$ \\
$\mathrm{~N}$ & 7.15 & \pm 0.25 & $1.41 \times 10^{-5}$ & $1.46 \times 10^{-4}$ \\
$\mathrm{O}$ & 6.82 & \pm 0.33 & $6.61 \times 10^{-6}$ & $7.79 \times 10^{-5}$ \\
$\mathrm{Ne}$ & 6.83 & \pm 0.30 & $6.76 \times 10^{-6}$ & $9.96 \times 10^{-5}$ \\
$\mathrm{~S}$ & $<5.5$ & & $<3.16 \times 10^{-7}$ & $<7.45 \times 10^{-6}$ \\
$\mathrm{Ar}$ & $<4.5$ & & $<3.16 \times 10^{-8}$ & $<8.38 \times 10^{-7}$ \\
\hline
\end{tabular}

photons increases with time, the ionization level of the dynamical model should be lower than that of the corresponding static model. Hence, Lyman alpha cooling should be more important and the electron temperature lower than in the hydrostatic model, which is indeed what their dynamical model yields. In TS 01 the dynamical effects on the ionization and temperature of the nebula should be much smaller than in the case computed by Schönberner et al., if noticeable at all. In their model, the temperature drop due to dynamical effects is about $10 \mathrm{kK}$. Given the argumentation above, we consider that any dynamical effect on the electron temperature in TS 01 would be of $2-3 \mathrm{kK}$ at most with respect to the temperature we compute in our model. As an experiment, we computed a model where we use the CLOUDY parameter "cextra" with a value of $10^{-20.3} \mathrm{erg} \mathrm{cm}^{3} \mathrm{~s}^{-1}$ to simulate an extra cooling factor that reduces the average electron temperature by about $3 \mathrm{kK}$ with respect to the reference model. We then adjusted the abundances to reproduce the observed line ratios. We found that the abundances in this model are not very different from those of model $\mathrm{R}$. In particular, the abundance of $\mathrm{O}$ is not changed. The reason is that the model must reproduce the [Ne V] $\lambda 3426 /[\mathrm{Ne} \mathrm{III]} \lambda 3869$ ratio, which is nearly temperatureindependent, and that ratios used to constrain the oxygen abundance ([O IV] $\lambda 25.9 \mu \mathrm{m} / \mathrm{H} \beta$ and [O III] $\lambda 5007 / \mathrm{H} \beta$ ) are not very sensitive to the temperature above $30 \mathrm{kK}$.

\subsection{Wrapping up}

In summary, considering all the possible sources of uncertainties and adding in quadrature the various independent errors, we find that the elemental abundances in the gas phase of TS 01 are as listed in Table 6.

An additional amount of carbon, about $40 \%$ of the total elemental abundance, is locked up in dust grains. Allowance for this component raises the carbon abundance in the nebula to $12+\log \mathrm{C} / \mathrm{H}=8.00 \pm 0.3$.

The error bars on the derived abundances may seem large when compared to the typical error bars in other PNe. However, one must remember that the analysis of TS 01 is much more difficult, due to the absence of direct temperature diagnostics and to the weakness of the lines from metals.

The abundances derived for the nebula are consistent with those derived by T. R. for the atmosphere of the cool star, see Sect. 3.3, except for carbon whose abundance is larger by 0.8 dex in the nebula. This agreement is remarkable, given the difficulty of the analysis. Whether the discrepancy between the carbon stellar and nebular abundances is real should be examined in more detail. 

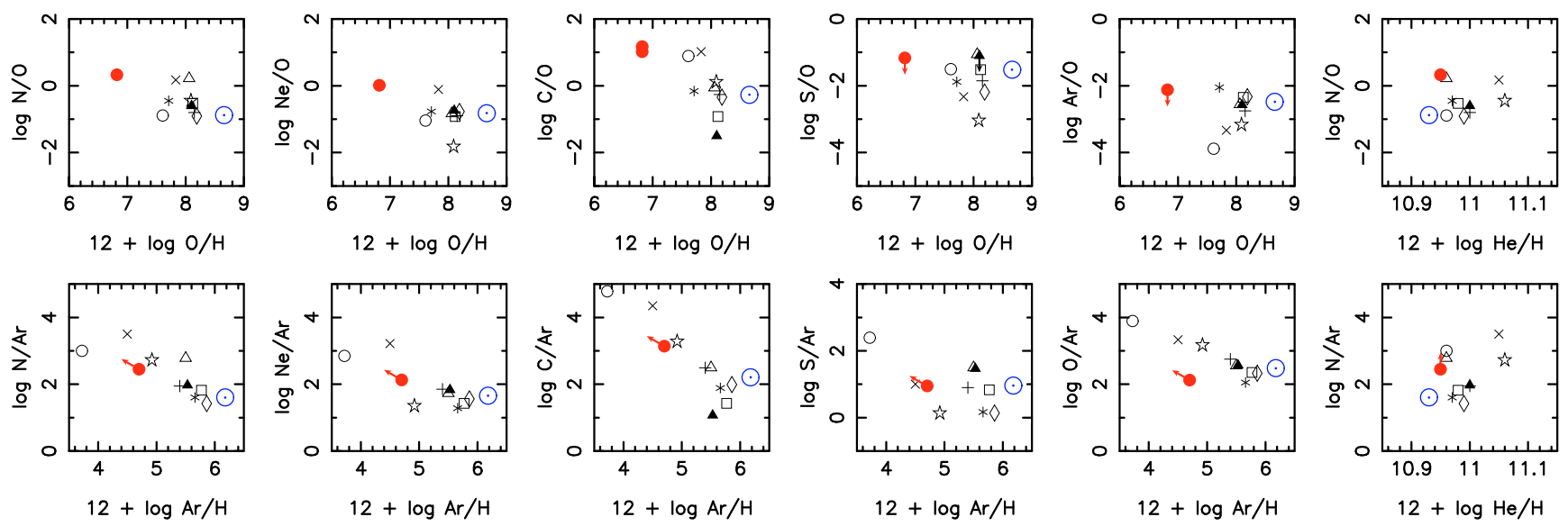

Fig. 17. Comparison of TS 01 (filled red circle) with other Galactic PNe in abundance ratios diagrams. The different black symbols represent: K648 (circle), DdDm-1 (square), PN006-41.9 (triangle), NGC 2242 (diamond), NGC 4361 (plus sign), M2-29 (asterisk), H4-1 (star), PN 243.837.1 (filled triangle), and BoBn-1, which actually belongs to the Sagittarius dwarf spheroidal galaxy ( $\times$ sign). The Sun is represented by the solar symbol.

\section{Understanding the observed chemical composition}

\subsection{TS 01 compared to other Galactic halo PNe}

Figure 17 shows the abundance pattern of TS 01 with respect to those of other PNe located in the Galactic halo as derived by Howard et al. (1997). The panels in the top rows of this figure show the elemental abundances with respect to oxygen as a function of $\mathrm{O} / \mathrm{H}$, a common way to display abundances in PNe. As has been noted before, PNe belonging to the Galactic halo display a large dispersion in their abundances relative to oxygen ${ }^{11}$. In this respect, TS 01 is not an exception. But it is extreme in its value of $\mathrm{O} / \mathrm{H}$ which is significantly lower than in other $\mathrm{PNe}$ of the Galactic halo. On the other hand, the $\mathrm{C} / \mathrm{O}, \mathrm{N} / \mathrm{O}, \mathrm{Ne} / \mathrm{O}$ ratios are similar to the highest ones found in those objects. In the panels of the bottom row, the abundance ratios are computed with respect to argon and displayed as a function of $12+\log \mathrm{Ar} / \mathrm{H}$. The reason for doing this is that the abundance of Ar is not expected to be modified with respect to the initial chemical composition out of which the progenitor star was formed. Of course, the determination of the argon abundance is much less accurate than that of oxygen. In the case of TS 01, we even have only an upper limit. Yet, the bottom row of Fig. 17 confirms the impression that, regarding the chemical composition, TS 01 is different from the remaining halo $\mathrm{PNe}$ especially because of its remarkable low oxygen abundance. It may have the lowest $\mathrm{Ar} / \mathrm{H}$ as well, but we do not know, since we have only an upper limit on its abundance.

As can be seen from Fig. 17, the progenitors of many of the PNe belonging to the Galactic halo underwent considerable nuclear processing, which affected not only the helium, carbon and nitrogen abundances in the nebulae, but also the oxygen one. In such a situation, the metallicity of the progenitor - generally identified with the oxygen abundance in the nebula - cannot be easily determined. If one uses the sum of the mass-fraction abundances of $\mathrm{C}, \mathrm{N}, \mathrm{O}, \mathrm{Ne}, \mathrm{S}$, and $\mathrm{Ar}$ as a proxy for the upper limit of the metallicity, we find that in solar units, TS 01 has a metallicity of at most 1/12 solar, well below the upper limits for the remaining halo PNe. If we take the argon abundance as a proxy for the metallicity, we find that the metallicity of TS 01 is less than $1 / 30$ solar. The two other halo PNe with very low metallicity, using

\footnotetext{
11 The dispersion in S/O, however, is likely significantly affected by important errors in the sulfur abundances.
}

this criterion, are BoBn-1 (1/20 solar) and K 648, which holds the record from this point of view (1/300 solar).

\subsection{What we know of the progenitor of TS 01}

Let us first summarize the main features of a possible evolution of the stellar core of TS 01 as explained in Tovmassian et al. (2007, 2008, and in prep.). The total mass of the system is close to $1.4 M_{\odot}$. The mass of the cool component is $(0.5-0.6) M_{\odot}$. Then the mass of the hot component must be $(0.8-0.9) M_{\odot}$. For $Z \simeq 0.001$ this implies that its progenitor had a mass of $\lesssim 3 M_{\odot}$ (Hurley et al. 2000). Star formation in the halo ceased at least 10 Gyr ago, see e.g. Marín-Franch et al. (2009). Since the lifetime of a $(2-3) M_{\odot}$ star with $Z \simeq 0.001$ is $\$ 300 \mathrm{Myr}$, the first Roche lobe overflow occurred at least $10 \mathrm{Gyr}$ ago. It resulted in a common envelope with inefficient accretion, hardly more than $\sim 0.01 M_{\odot}$ (Yungelson et al. 1995). The initially more massive star of the system turned into the currently hot component. Since we observe a PN now, the companion of the hot component must have completed its evolution in $10 \mathrm{Gyr}$. The least massive star able to do this had a mass of about $0.9 M_{\odot}$ after the first common envelope stage. When this low mass star evolved off the main sequence, a symbiotic system formed. Mass transfer during this stage was negligible as well. The symbiotic stage ended when the low mass star filled its Roche lobe on the AGB. Again, a common envelope formed, reducing the separation of the components and diminishing the orbital period to $3.9 \mathrm{~h}$. The nebula we observe now is the remnant of this second common envelope.

\subsection{Can standard single AGB models explain the observed abundance pattern?}

We consulted the available yields from recent standard evolution models for single stars at low metallicities (see Karakas \& Lattanzio 2003; and Cristallo et al. 2009). The corresponding nucleosynthetic predictions for low-mass AGBs show the signature of recurring third dredge-up episodes that increase the surface abundances of $\mathrm{C}$ as well as (but to a lower extent) that of $\mathrm{O}$ and $\mathrm{N}$. This is at odds with the abundance patterns determined in TS 01, which exhibits an extreme $\mathrm{O}$ depletion. As a matter of fact, only massive AGB models $\left(M_{\text {ini }} \geq 4 M_{\odot}\right)$ predict $\mathrm{O}$ depletion as a result of hot bottom burning (HBB) during the thermal pulse AGB phase (TP-AGB). Besides being in conflict with 
the binary analysis recalled above, these massive AGB models are facing other difficulties. First, they predict that carbon also should be depleted by the CNO-cycle in the envelope. Second, such massive AGB stars are predicted to experience a deep second dredge-up that increases the He abundance well above the observed value (number ratio of $\mathrm{He} / \mathrm{H} \sim 0.093$, thus $\mathrm{Y} \sim 0.26$ in mass fraction).

\subsection{Towards an explanation of the chemical composition of TS 01}

We note however that those models use standard assumptions. In particular they do not account for rotation-induced mixing that is known to affect stellar evolution and nucleosynthesis (see Decressin et al. 2009b and references therein). Additionally, the rotational transport itself may have been strengthened by the stellar coupling in the binary system (see e.g. de Mink et al. 2009; Pols et al. 1997). Unfortunately binary stellar models which treat the effect of mixing on the nucleosynthesis of lowmass stars are not yet available, but we can use single rotating AGB models to infer the impact of mixing during the pre-AGB phase on the surface chemical composition.

We thus compare the abundance obtained for our nebula to standard and rotating models of a $1 M_{\odot}$ star computed with the code STAREVOL (V2.92) (Siess et al. 2000; Siess 2006) from the pre-main sequence to the end of the TP-AGB phase (for more details, see Decressin et al. 2009a). In the rotating model, an initial rotation of $100 \mathrm{~km} \mathrm{~s}^{-1}$ is assumed on the zero age main sequence. This is the mean value given by Gaige (1993) for a star with the effective temperature of our models near the ZAMS $(\sim 7000 \mathrm{~K})$. This observational value is obtained from stars in the Hyades, which are more metal-rich than our model. If low-metallicity stars are born with the same amount of angular momentum we could expect, due to their compactness, an even higher initial velocity. Thus our model indicates the minimal chemical changes we would obtain when rotation is taken into account.

The transport of angular momentum and chemical species is driven by meridional circulation and shear turbulence (Zahn 1992; Maeder \& Zahn 1998). The metallicity is set to $Z=$ $5 \times 10^{-4}$ (i.e., $\left.[\mathrm{Fe} / \mathrm{H}] \simeq-1.6\right)$, which corresponds to the upper limit for the metallicity derived from the Ar abundance in TS 01 . The composition is scaled to the solar one according to the Grevesse \& Sauval (1998) mixture, and enhancement in $\alpha$ elements $([\alpha / \mathrm{Fe}]=+0.3 \mathrm{dex})$ is accounted for. We use the OPAL opacity tables (Iglesias \& Rogers 1996) above a temperature of $8 \mathrm{kK}$ that account for $\mathrm{C}$ and $\mathrm{O}$ enrichments, and the Ferguson et al. (2005) data at lower temperatures. We follow the evolution of 53 chemical species from ${ }^{1} \mathrm{H}$ to ${ }^{37} \mathrm{Cl}$. We use the NACRE nuclear reaction rates (Angulo et al. 1999) by default and those by Caughlan \& Fowler (1988) otherwise (see Siess \& Arnould 2008). The treatment of convection is based on the classical mixing length formalism with $\alpha_{\mathrm{MLT}}=1.75$, and diffusive overshoot is added below the convective envelope according to Herwig (2000). The mass loss rate is computed with the Reimers (1975) formula (with $\eta_{\mathrm{R}}=0.5$ ) for the pre-AGB phase and then we use the Vassiliadis \& Wood (1993) one during the AGB phase. We also add diffusive overshoot following Herwig (2000) to allow for a third-dredge up during the AGB phase.

When comparing the results from rotating AGB models with those of non-rotating ones, we find considerable differences in the predictions of the chemical composition as illustrated by Fig. 18. In particular rotation-induced mixing efficiently transports chemical species from the H-burning shell to the surface
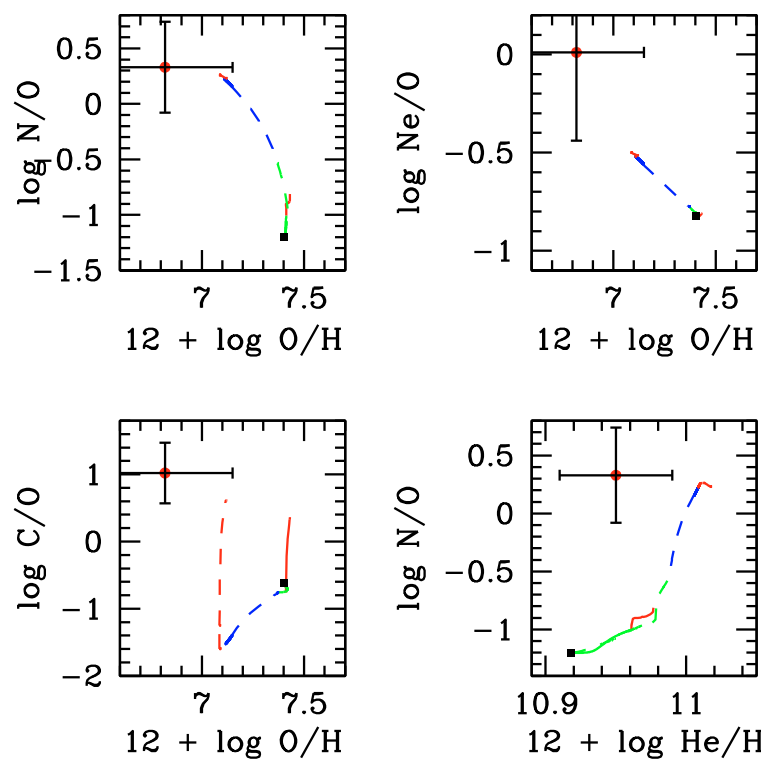

Fig. 18. Comparison of the chemical composition in TS 01 with the results of stellar evolution models for a star with an initial mass $1 M_{\odot}$, and an initial composition as indicated by the black dot. The model without rotation is represented with a continuous line, the model with rotation is represented with dotted lines. The modifications of the chemical composition on the main sequence are indicated in green, those during the second dredge-up are in blue, and those corresponding to the TP-AGB phase in red.

during central He-burning. This tends to decrease the surface abundances of $\mathrm{C}$ and $\mathrm{O}$ in favour of that of $\mathrm{N}$ through the CNOcycle while He increases only mildly. As can be seen in Fig. 18, rotation-induced mixing leads to low $\mathrm{O}$ and high $\mathrm{N}$ abundances already at the end of the second dredge-up. From Fig. 18, we see that introducing rotation (and thus strong internal mixing which can mimick binary interactions) dramatically improves the agreement of the predicted abundances with the chemical composition of TS 01 . Still, the O depletion in the model is consistent only with the upper limit allowed by the observations. The agreement would be improved if the star rotates faster during the phase of central $\mathrm{H}$ - and He-burning so that more efficient mixing induces stronger $\mathrm{O}$ depletion and $\mathrm{N}$ enhancement. As already explained above the chosen initial velocity for our models could be slightly too low for its metallicity. Furthermore an even more pronounced rise of the rotational rate can be the result of the first mass transfer when the massive primary star expands. This high velocity can also lead to a stronger enhancement of $\mathrm{Ne}$ as required by the observations. In this way the full observational pattern of TS 01 can be explained, except for the He abundance which is still higher than observed.

\section{Conclusions}

The planetary nebula PN G 135.9+55.9 (SBS 1150+599A), here referred to as TS 01 , has been a source of many surprises. It is the most oxygen-poor planetary nebula known. Its stellar core consists of a close binary, whose period of approximately $3.9 \mathrm{~h}$ is the shortest known of any planetary nebula nucleus. The binary nucleus is the first known example of a double degenerate system in a planetary nebula. It is also the best candidate progenitor of a type Ia supernova. Here we consider the chemical composition of the nebular shell in detail. In a companion paper, Tovmassian et al. (in prep.) study the nature, history, and evolution of the binary system. 
We have obtained observational data in a complete wavelength range in order to pin down the abundances of half a dozen of elements in TS 01. The data consist of imaging and spectroscopy in the UV, optical and IR ranges. Optical data were obtained at different telescopes, resulting in high quality and reliable observational material for this object which exhibits an exceptionally line-poor optical spectrum.

We then defined a strategy to determine the abundances in TS 01. Since no direct electron temperature diagnostic is available, it was necessary to apply tailored photoionization modelling. We did this with much care, taking into account all the observational information at our disposal, in order to minimize the uncertainties in the derived abundances. The photoionization modelling was performed using the pseudo-3D photoionization code Cloudy_3D based on CLOUDY (version c07.02.01). Using CLOUDY, we took advantage of its detailed treatment of the hydrogen atom, which is crucial in this density-bounded nebula. Using the 3D features allowed us to take into account the apparent geometry of the nebula and the location, sizes and orientations of the observing slits, thus minimizing the errors linked to aperture effects. The spectral energy distribution of the ionizing radiation was obtained from appropriate model atmospheres.

One interesting feature of TS 01 is that both its stellar components contribute to its ionization: the "cool" one provides the bulk of hydrogen ionization, and the "hot" one is responsible for the presence of the most highly charged ions. Thus one can say that TS 01 has two ionizing stars. This is the first such case discovered among planetary nebulae!

The abundances of $\mathrm{C}, \mathrm{N}, \mathrm{O}$, and $\mathrm{Ne}$ in the nebula are found to be $1 / 3.5,1 / 4.2,1 / 70$, and $1 / 11$ of the solar value, respectively, with error bars of \pm 0.3 dex. For $S$ and Ar, the abundances are less than $1 / 30$ of solar. This makes of TS 01 the planetary nebula with the most extreme composition known so far. In particular, it is by far the most oxygen-deficient. The abundance of helium is $12+\log \mathrm{He} / \mathrm{H}=10.95$, with an accuracy that is not as high as one might have desired $( \pm 0.04 \mathrm{dex})$. The nebular abundances in TS 01 are found to be consistent with those in the atmosphere of the cool star, except for carbon which appears higher by $\sim 0.8 \mathrm{dex}$ than in the star.

The observed properties of the stellar core allow us to put limits on the masses and age of the binary system, as explained in Tovmassian et al. (in prep.) and recalled in the present paper. In particular, the initial mass of the progenitor of the present nebula was $\sim 0.9 M_{\odot}$. We have computed state-of-the-art AGB models with appropriate mass and metallicity to compare them with the chemical composition of TS 01 . While a non-rotating model cannot explain the observed abundance patterns, the introduction of a $100 \mathrm{~km} \mathrm{~s}^{-1}$ initial rotation in the model (which may be seen as a way to mimick the mixing due to the presence of the companion) greatly improves the comparison. Rotationinduced mixing leads to low $\mathrm{O}$ and high $\mathrm{N}$ abundances already at the end of the second dredge-up, whereas the $\mathrm{C}$ abundance increases afterwards during each third dredge-up. However, to fully reproduce the observed pattern requires the binary interactions (and especially the first episode of mass transfer) to spin up the star to produce a very efficient mixing. This conjecture still needs to be verified by proper binary models.

One feature for which we have no explanation so far is the fact that the carbon abundance in the nebula seems to be much higher than in its progenitor. This fact would need to be confirmed with deeper observations in the optical and the UV though.

With respect to our first publication on TS 01 by Tovmassian et al. (2001), our understanding of the status and properties of this object has thus made enormous progress. While this object is unique when considering all its observed properties together, similar objects might be plentiful in the Galactic halo although not necessarily in a stage exhibiting a detectable planetary nebula.

Acknowledgements. This work, which extended over a period of more than seven years, could be accomplished thanks to extensive use of e-mail, short visits and informal meetings on the occasion of conferences. We thank Gary Ferland and his associates for having offered CLOUDY to the community. We are indebted to A. Weiss for providing unpublished evolutionary tracks. We are grateful to Yuri Izotov for sending the TS 01 spectrum he obtained at Kitt Peak and for drawing our attention to the existence of this object in the SDSS data base (he found it while examining by eye the hundreds of thousands of spectra from the main galaxy sample!). Funding for the Sloan Digital Sky Survey (SDSS) has been provided by the Alfred P. Sloan Foundation, the Participating Institutions, the National Science Foundation, the US Department of Energy, the National Aeronautics and Space Administration, the Japanese Monbukagakusho, the Max Planck Society, and the Higher Education Funding Council for England. G.S. and S.S.-D. acknowledge the hospitality and financial support of the Instituto de Astronomia of the UNAM in Mexico City and Ensenada during many visits. C.M. acknowledges the hospitality and support of the Observatoire de Paris and the grant CONACyT-49749. G.S., C.M., R.Sz, R.N., S.S.-D. acknowledge support from the European Associated Laboratory "Astrophysics Poland-France". G.T. acknowledges a grant from UC-MEXUS, which made his stay at CASS UCSD possible and continuous support from CONACyT. T.R. is supported by the German Federal Ministry of Education and Research (BMBF) grant 50 OR 0806. M.R. acknowledges the following grants: CONACyT 43121 and 82066, DGAPA-UNAM IN116908, 112103, 108506, and 108406. M.P. acknowledges financial support from UNAM-DGAPA grants IN118405 and IN112708 and from a CONACyT-CNRS 2008 project. R.Sz. acknowledge partial support from grant N203 393334 of MNiSW/Poland. C.C. and T.D. acknowledge financial support from the French Programme National de Physique Stellaire (PNPS) of CNRS/INSU, and from the Swiss National Science Foundation (FNS). L.Yu. acknowledges support from RFBR grant 07-02-00454 and Presidium of the Russian Academy of Sciences Program "Origin, Evolution and Structure of the Universe Objects". S.S.-D. acknowledges support from the Spanish Ministerio de Educación y Ciencia under the MEC/fulbright postdoctoral program.

Note added in proof. Sandin et al. (arXiv:0912.5430) recently derived the nebular abundances in PN G 135.9+55.9 using hydrodynamical models, and claim that in this object, departure from thermal equilibrium leads to lower electron temperatures and hence weaker emission in collisionally excited lines. The abundances they give, however, turn out to be similar to the ones we find within the error bars that we provide (and which actually take into account the uncertainty due to dynamical effects). The reason for this similarity is partly fortuitous. Briefly, the observational constraints they consider are not exactly the same as ours. In particular, these authors do not correct the C IV $\lambda 1549$ line for absorption, nor do they consider depletion unto dust grains, they do not take into account aperture effects, and they do not compare their computed infrared line fluxes to observed ones. On the other hand, the scenario they adopt for their hydrodynamical model (a single star with mass $0.595 M_{\odot}$ ) contradicts our observations of the stellar core (see Tovmassian et al. 2007, and Sects. 3 and 6 of our paper). As explained in Sect. 5.3 of our paper, the dynamical effects in PN G 135.9+55.9 must be less important than they claim.

\section{References}

Acker, A., Marcout, J., Ochsenbein, F., Stenholm, B., \& Tylenda, R. 1992, Strasbourg - ESO catalogue of galactic planetary nebulae. Part 1; Part 2 (Garching: European Southern Observatory)

Adelman-McCarthy, J. K., Agüeros, M. A., Allam, S. S., et al. 2008, ApJS, 175, 297

Angulo, C., Arnould, M., Rayet, M., et al. 1999, Nuclear Phys. A, 656, 3 
Asplund, M., Grevesse, N., \& Sauval, A. J. 2005, in Cosmic Abundances as Records of Stellar Evolution and Nucleosynthesis, ed. T. G. Barnes, III, \& F. N. Bash, ASP Conf. Ser., 336, 25

Baker, J. G., \& Menzel, D. H. 1938, ApJ, 88, 52

Bouchet, P., Lequeux, J., Maurice, E., Prevot, L., \& Prevot-Burnichon, M. L. 1985, A\&A, 149, 330

Caughlan, G. R., \& Fowler, W. A. 1988, Atomic Data and Nuclear Data Tables, 40,283

Cristallo, S., Straniero, O., Gallino, R., et al. 2009, ApJ, 696, 797

de Mink, S. E., Cantiello, M., Langer, N., et al. 2009, A\&A, 497, 243

Decressin, T., Charbonnel, C., Siess, L., et al. 2009a, A\&A, 505, 727

Decressin, T., Mathis, S., Palacios, A., et al. 2009b, A\&A, 495, 271

Ferguson, J. W., Alexander, D. R., Allard, F., et al. 2005, ApJ, 623, 585

Ferland, G. J. 1999, PASP, 111, 1524

Ferland, G. J., Korista, K. T., Verner, D. A., et al. 1998, PASP, 110, 761

Filippenko, A. V. 1982, PASP, 94, 715

Fitzpatrick, E. L. 1999, PASP, 111, 63

Gaige, Y. 1993, A\&A, 269, 267

Grevesse, N., \& Sauval, A. J. 1998, Space Sci. Rev., 85, 161

Herwig, F. 2000, A\&A, 360, 952

Higdon, S. J. U., Devost, D., Higdon, J. L., et al. 2004, PASP, 116, 975

Houck, J. R., Roellig, T. L., van Cleve, J., et al. 2004, ApJS, 154, 18

Howard, J. W., Henry, R. B. C., \& McCartney, S. 1997, MNRAS, 284, 465

Hurley, J. R., Pols, O. R., \& Tout, C. A. 2000, MNRAS, 315, 543

Iglesias, C. A., \& Rogers, F. J. 1996, ApJ, 464, 943

Jacoby, G. H., Feldmeier, J. J., Claver, C. F., et al. 2002, AJ, 124, 3340

Jacoby, G. H., Garnavich, P. M., Bond, H. E., et al. 2006, in Planetary Nebulae in our Galaxy and Beyond, ed. M. J. Barlow, \& R. H. Méndez, IAU Symp., 234,431

Karakas, A. I., \& Lattanzio, J. C. 2003, PASA, 20, 279

Landi, E., Del Zanna, G., Young, P. R., et al. 2006, ApJS, 162, 261

Le Fèvre, O., Crampton, D., Felenbok, P., \& Monnet, G. 1994, A\&A, 282, 325

Maeder, A., \& Zahn, J.-P. 1998, A\&A, 334, 1000

Marín-Franch, A., Aparicio, A., Piotto, G., et al. 2009, ApJ, 694, 1498

McCall, M. L., Rybski, P. M., \& Shields, G. A. 1985, ApJS, 57, 1
Morisset, C. 2006, in Planetary Nebulae in our Galaxy and Beyond, ed. M. J. Barlow, \& R. H. Méndez, IAU Symp., 234, 467

Napiwotzki, R., Tovmassian, G., Richer, M. G., et al. 2005, in Planetary Nebulae as Astronomical Tools, ed. R. Szczerba, G. Stasinska, \& S. K. Gorny, AIP Conf. Ser., 804, 173

Péquignot, D., \& Tsamis, Y. G. 2005, A\&A, 430, 187

Pols, O. R., Tout, C. A., Schroder, K.-P., Eggleton, P. P., \& Manners, J. 1997, MNRAS, 289, 869

Prévot, M. L., Lequeux, J., Prevot, L., Maurice, E., \& Rocca-Volmerange, B. 1984, A\&A, 132, 389

Rauch, T. 2003, A\&A, 403, 709

Reimers, D. 1975, Circumstellar envelopes and mass loss of red giant stars (Problems in stellar atmospheres and envelopes), 229

Richer, M. G., López, J. A., Steffen, W., et al. 2003, A\&A, 410, 911

Richer, M. G., Tovmassian, G., Stasińska, G., et al. 2002, A\&A, 395, 929

Schlegel, D. J., Finkbeiner, D. P., \& Davis, M. 1998, ApJ, 500, 525

Schönberner, D., Jacob, R., Steffen, M., \& Roth, M. M. 2005, in Planetary Nebulae as Astronomical Tools, ed. R. Szczerba, G. Stasińska, \& S. K. Gorny, AIP Conf. Ser., 804, 269

Siess, L. 2006, A\&A, 448, 717

Siess, L., \& Arnould, M. 2008, A\&A, 489, 395

Siess, L., Dufour, E., \& Forestini, M. 2000, A\&A, 358, 593

Stepanian, J. A., Chavushyan, V. H., Carrasco, L., Tovmassian, H. M., \& Erastova, L. K. 1999, PASP, 111, 1099

Storey, P. J., \& Hummer, D. G. 1995, MNRAS, 272, 41

Tovmassian, G., Tomsick, J., Napiwotzki, R., et al. 2007 [arXiv:0709.4016]

Tovmassian, G., Tomsick, J., Napiwotzki, R., et al. 2008, in Astrophysics of Compact Objects, ed. Y.-F. Yuan, X.-D. Li, \& D. Lai, AIP Conf. Ser., 968, 62 Tovmassian, G. H., Stasińska, G., Chavushyan, V. H., et al. 2001, A\&A, 370, 456

Tovmassian, G. H., Napiwotzki, R., Richer, M. G., et al. 2004, ApJ, 616, 485

Vassiliadis, E., \& Wood, P. R. 1993, ApJ, 413, 641

Werner, M. W., Roellig, T. L., Low, F. J., et al. 2004, ApJS, 154, 1

Yungelson, L., Livio, M., Tutukov, A., \& Kenyon, S. J. 1995, ApJ, 447, 656

Zahn, J.-P. 1992, A\&A, 265, 115 